\title{
Topological T-duality and Kaluza-Klein monopoles
}

\author{
Ashwin S. Pande ${ }^{\dagger}$ \\ Department of Mathematics, University of Maryland at College Park, \\ College Park, MD 20742, USA \\ asp105@rsphysse.anu.edu.au
}

\begin{abstract}
We study topological T-duality for spaces with a semi-free $S^{1}$-action with isolated fixed points. Physically, these correspond to spacetimes containing Kaluza-Klein monopoles. We demonstrate that the physical dyonic coordinate of such spaces has an analogue in our formalism. By analogy with the Dirac monopole, we study these spaces as gerbes. We study the effect of topological T-duality on these gerbes.
\end{abstract}

\section{Introduction}

String theory replaces point particles by extended objects called strings propagating in a background spacetime called the "Target Space" [1]. Torus duality or T-duality in string theory is an important symmetry of string theories in which the target space has a torus action: IIA string theory with a target spacetime $X$ possessing a $T^{n}$-action is identical to IIB string theory on the T-dual spacetime $X^{\#}[2]$; i.e., the two quantum theories are identical up to a canonical transformation.

\footnotetext{
${ }^{\dagger}$ Current address: Theoretical Physics, RSPhysSE, ANU, Canberra, ACT 2602. e-print archive: http://lanl.arXiv.org/abs/arXiv:math-ph/0612034
} 
In the case that spacetime is a principal $T^{n}$ bundle over a base, the dual spacetime $X^{\#}$ is roughly one in which each torus orbit in the original spacetime has been replaced by its dual torus (following Ref. [3], if $\Lambda$ is a lattice in $\mathbb{R}^{n}$ and $\Lambda^{*}$ is the dual lattice in the dual vector space $\left(\mathbb{R}^{n}\right)^{*}$, then the torus dual to $\mathbb{R}^{n} / \Lambda$ is $\left.\left(\mathbb{R}^{n}\right)^{*} / \Lambda^{*}\right)$. In the original string theory calculation, the T-dual is obtained as a manifold together with various extra data (metric, B-field, dilaton, and R-R charges). ${ }^{1}$

Surprisingly, if we restrict ourselves to spacetimes which are principal $T^{n}(n=1,2)$ bundles over some base, it is possible to develop a theory of T-duality using only topological information. ${ }^{2}$ That is, it is possible to specify the topological structure of the T-dual of a given spacetime using only the $H$-flux and topological structure of the original spacetime. ${ }^{3}$ This is surprising since string theory usually requires a smooth, semi-Riemannian manifold (usually a Kähler manifold) as its target. The resulting theory has been the subject of the papers $[3,4,5]$ and may be viewed as a "topological approximation" to T-duality in string theory. That is, it should be possible to take a spacetime $X$ which possesses a T-dual in the sense of Ref. [3] and give it additional structure (metric, spin etc.) so as to construct on $X$ a type IIA string theory and to construct on $X^{\#}$ a type IIB string theory which form a dual pair (however, see Ref. [5]).

Suppose $X$ is homeomorphic to a smooth closed, connected Riemannian manifold. Suppose, further that $X$ possesses a smooth, semi-free, action of $T^{n},(n=1,2)$. (By a semi-free action, we mean an action with exactly two types of orbits: free orbits and fixed points.) Then we are in the basic setup of Ref. [3] if a class $\delta \in H^{3}(X, \mathbb{Z})$ is specified. Here $X$ is to be viewed as a spacetime and the map $X \rightarrow X / T^{n}$ is a degenerate fibration.

If the semi-free action has no fixed points, it is free and so by the Gleason slice theorem, $X$ is a principal $T^{n}$-bundle over $X / T^{n}$. This case has already been extensively studied in Refs. $[3,6]$. There, the authors associate to a space $X$ with $H$-flux $\delta$, the continuous trace algebra $\operatorname{CT}(X, \delta)$. If $X$ is a principal $T^{n}$-bundle $(n=1,2)$, they demonstrate the following:

- If $n=1$, there is a unique lifting $\alpha$ of the $\mathbb{R}$-action on $X$ to $\mathrm{CT}(X, \delta)$. The T-dual spacetime to $X$ is given by the spectrum of $\mathrm{CT}(X, \delta) \underset{\alpha}{\rtimes} \mathbb{R}$. In this case, the crossed product is always continuous trace.

\footnotetext{
${ }^{1}$ It was the study of the mapping of the R-R charges of string theory under such a transformation that gave the initial impetus to study T-duality purely topologically [4].

${ }^{2}$ The metric structure and finer structures (like the Kahler structure, needed for supersymmetry) are not needed to determine the topological type of the T-dual.

${ }^{3}$ Here "topological structure" should be interpreted broadly because the T-dual may be a non-commutative space.
} 
- If $n=2$ and if a certain condition is satisfied, the T-dual is unique and is given by the spectrum of the crossed product as above. If this condition is violated, however, there is no unique lifting of the $\mathbb{R}^{n}$ action on $X$ to $\operatorname{CT}(X, \delta)$. Also, the crossed product is not Type $\mathrm{I}$. However, as explained in Ref. [3], the T-dual may be viewed as a non-commutative space.

In either case, the natural action of $\hat{\mathbb{R}}^{n}$ on the spectrum of the crossed product makes it into a principal $T^{n}$-bundle over $X / \mathbb{R}^{n}$.

In this paper, we study the T-duals of semi-free $S^{1}$-spaces. In Section 2, we show that the formalism of Ref. [3] extends to spaces containing KKmonopoles. In Section 3, we show that the result obtained in the previous section may be used to define an analogue of the dyonic coordinates of a system of KK-monopoles within the formalism of topological T-duality. In Section 4, we study the result of Section 2 using gerbes.

\section{Kaluza-Klein monopoles and T-duality}

In this paper, we study semi-free $S^{1}$-spaces with non-empty fixed point sets. Now, it is well known [7] that a smooth action of a compact Lie group $G$ on a smooth manifold $M$ in a neighborhood of a fixed point is equivariantly- $G$ homeomorphic to an orthogonal action of $G$ on a finite-dimensional vector space. It would be helpful to consider this case first.

Thus, now we let $X$ be $\mathbb{R}^{k}$ with a faithful orthogonal action of $S^{1}$. We view this as defining a fibration of $\mathbb{R}^{k}$ over the quotient space. We attempt to define a T-dual for such a fibration. Note that there can be no $H$-flux in this setting, since $\mathbb{R}^{k}$ is contractible (i.e., $\delta=0$ ). As in Ref. [3], we introduce the $C^{*}$-algebra $\mathcal{A}=\mathrm{CT}(X, \delta)$. We lift the $S^{1}$-action on $X$ to a $\mathbb{R}^{n}$-action $\alpha$ on $\mathcal{A}$. This may always be done for all $n$ because $X$ is contractible (see Raeburn and Rosenberg [6]. $)^{4}$ As in Ref. [3], we may attempt to define the T-dual of $X$ as the spectrum of the crossed product $\mathcal{A} \underset{\alpha}{\rtimes} \mathbb{R}$.

As a test example, let $X=\mathbb{C}^{2}$ with the $S^{1}$-action

$$
\mathrm{e}^{2 \pi \mathrm{i} \theta} \cdot(z, w)=\left(\mathrm{e}^{2 \pi \mathrm{i} \theta} z, \mathrm{e}^{2 \pi \mathrm{i} \theta} w\right) .
$$

This may be lifted to the obvious action of $\mathbb{R}$ namely,

$$
\alpha_{t}(z, w)=\left(\mathrm{e}^{2 \pi \mathrm{i} t} z, \mathrm{e}^{2 \pi \mathrm{i} t} w\right) .
$$

\footnotetext{
${ }^{4}$ For other spaces, if $n>1$, and $H^{3}(X, \mathbb{Z}) \neq 0$ Mackey obstructions may arise which prevent this lifting.
} 
Note that the $S^{1}$-action leaves each 3 -sphere

$$
S_{r}^{3}=\left\{\left.\left(z_{1}, z_{2}\right)|| z_{1}\right|^{2}+\left|z_{2}\right|^{2}=r^{2}\right\}
$$

invariant. The $S^{1}$-action on each $S_{r}^{3}$ has $S^{2}$ as quotient. The quotient map is the Hopf fibration. The origin $(0,0)$ is a fixed point for the $\mathbb{R}$-action.

We may also view this as a fibration of $C^{0} S^{3}$ over the base $C^{0} S^{2}$ (here, $\left.C^{0} S^{3} \simeq\left(S^{3} \times \mathbb{R}^{+}\right) /\left(S^{3} \times 0\right)\right)$ the open cone on $\left.S^{3}\right)$. The map sending $C^{0} S^{3}$ to $C^{0} S^{2}$ is the Hopf fibration when restricted to $S^{2} \times\{t\}, t \neq 0$ and sends the vertex of $C^{0} S^{3}$ to the vertex of $C^{0} S^{2}$. (Note $S^{3} \times(0, \infty)$ is a principal $S^{1}$ bundle over $S^{2} \times(0, \infty)$.)

\subsection{Physical T-duals}

As we mentioned in the introduction, T-duality was first discovered in the theory of closed strings. This formalism only allows us to calculate the T-dual of space with a free $T^{n}$-action. It was soon realized that string theory contains a theory of extended objects called "branes". These are (roughly) submanifolds of spacetime on which strings can end. Due to the strings ending on such a submanifold, ${ }^{5}$ there is a quantum field theory defined on it. There are two types of branes: the $\mathrm{D} p$-branes are submanifolds which are sources of the R-R fields of string theory; ${ }^{6}$ the NS5-branes, on the other hand are submanifolds which are sources of the Neveau-Schwartz-B-field. ${ }^{7}$ After the introduction of $\mathrm{D} p$-branes, T-duality was studied by putting $\mathrm{D} p$-brane probes into a geometry. The T-dual is the moduli space of the worldvolume theory on the $\mathrm{D} p$-brane. This approach is extremely flexible and enables the calculation of several T-duals unobtainable by previous methods.

It is useful to study the T-duals of semi-free $S^{1}$-spaces in the physics literature.

1) The T-dual of a NS5-brane is a Kaluza-Klein (KK) monopole (see Refs. [8-10]). Geometrically, an NS5-brane is a six-dimensional submanifold of $X$ which is a source of $H$-flux. Topologically, $X$ is a fibration $\mathbb{R}^{6} \times C^{0} S^{2} \times S^{1} \stackrel{\pi}{\rightarrow} \mathbb{R}^{6} \times C^{0} S^{2}$, where $\pi$ is the projection map. The worldvolume of the NS5-brane intersects the $S^{1}$-fiber $\pi^{-1}(0)$ at a

\footnotetext{
${ }^{5}$ The submanifold associated with a brane is termed its "worldvolume".

${ }^{6} \mathrm{D} p$-branes possess $(p+1)$-dimensional worldvolumes. In type IIA string theory, $p$ can only be even dimensional. In type IIB string theory, $p$ can only be odd dimensional. Their worldvolume theory at low energies is a Super-Yang-Mills gauge theory.

${ }^{7}$ These have six-dimensional worldvolumes. Their worldvolume theory is a string theory (a "little string theory"). At low string coupling, they are extremely massive compared to $\mathrm{D} p$-branes.
} 
single point while its six worldvolume directions occupy $\mathbb{R}^{6}$. Since $\mathbb{R}^{6}$ is contractible, it does not affect the topological type of the T-dual. In the following, we will model this by studying $C^{0} S^{2} \times S^{1} \stackrel{\pi}{\rightarrow} C^{0} S^{2}$. We will say that the NS5-brane is sitting at some location on the $S^{1}$ fiber over 0 . This brane emits 1 unit of $H$-flux which we model as the cohomology class [1] $\in H^{3}\left(C S^{2} \times S^{1}-\{0\} \times S^{1}\right)$, where [1] is the generator of

$$
H^{3}\left(S^{2} \times S^{1}\right) \simeq H^{3}\left(S^{2} \times(0, \infty) \times S^{1}\right) \simeq H^{3}\left(C^{0} S^{2} \times S^{1}-\pi^{-1}(0)\right) .
$$

A Kaluza-Klein monopole is a semi-Riemannian manifold, ${ }^{8}$ which solves Einstein's equations. Topologically, this manifold is $\mathbb{R}^{5}$ with metric $g_{\mathrm{KK}}=-d t^{2}+g_{\mathrm{TN}}$. Here $g_{\mathrm{TN}}$ is a certain Riemannian metric on $\mathbb{R}^{4}$ called the Taub-NUT metric. ${ }^{9}$ The space $\left(\mathbb{R}^{4}, g_{\mathrm{TN}}\right)$ (termed "TaubNUT" space) possesses an isometric action of $S^{1}$ with one fixed point, and is $S^{1}$-equivariantly homeomorphic to $\mathbb{C}^{2}$ with the $S^{1}$-action $\lambda$. $\left(z_{1}, z_{2}\right)=\left(\lambda \cdot z_{1}, \lambda \cdot z_{2}\right), \lambda \in S^{1} \subseteq \mathbb{C}, z_{i} \in \mathbb{C}$. In the physics literature, the time direction is often ignored and $\left(\mathbb{R}^{4}, g_{\mathrm{TN}}\right)$ is also called the "Kaluza-Klein monopole". For our purposes, a Kaluza-Klein monopole is an $S^{1}$ fibration over $\mathbb{R}^{3} \simeq C^{0} S^{2}$. Over each sphere $\{t\} \times S^{2}$ in the base, the fibration is the Hopf fibration. Over 0 in the base, the fibration degenerates to a point. It may be viewed as a fibration $C^{0} S^{3} \stackrel{\pi^{\#}}{\rightarrow} C^{0} S^{2}$.

Thus, we say that the string-theoretic T-dual of $C^{0} S^{3} \stackrel{\pi^{\#}}{\rightarrow} C^{0} S^{2}$ is $C^{0} S^{2} \times S^{1} \stackrel{\pi}{\rightarrow} C^{0} S^{2}$ together with the $H$-flux emitted from a point source sitting at some point on the fiber $\pi^{-1}(0)$ (also termed a $H$-monopole). $\pi^{\#}: C^{0} S^{3} \rightarrow C^{0} S^{2}$ is the test case discussed in Section 2.2. It is an important example, because (as will be seen below) most physical examples are built up from this one.

This T-dual may be calculated using Buscher's rules. We use polar coordinates $(r, \theta, \phi)$ to parametrize the base and a periodic coordinate $\kappa$ to parametrize the $S^{1}$-fiber. Following Ref. [11], the Riemannian metric on Taub-NUT space may be written as

$$
g_{\mathrm{TN}}=H(r) d \vec{r} \cdot d \vec{r}+H(r)^{-1}\left(d \kappa+\frac{1}{2} \vec{\omega} \cdot d \vec{r}\right)^{2},
$$

where $d \vec{r}=(d r, d \theta, d \phi)$, (note that $\phi$ is the periodic polar coordinate). $H(r)=g^{-2}+(2 r)^{-1}$, and $\omega_{r}=\omega_{\theta}=0, \omega_{\phi}=(1-\cos (\theta))$. We may

\footnotetext{
${ }^{8}$ see Refs. [10-12].

${ }^{9}$ This is an example of an ALF gravitational instanton metric.
} 
write

$$
\begin{aligned}
g_{\mathrm{TN}}= & H d r^{2}+H r^{2} \sin ^{2}(\theta)(d \phi)^{2}+H r^{2}(d \theta)^{2}+H^{-1}(d \kappa)^{2} \\
& +H^{-1}(1-\cos (\theta)) d \phi d \kappa+H^{-1}(1-\cos (\theta))^{2}(d \phi)^{2},
\end{aligned}
$$

where $\kappa$ is the coordinate on the $S^{1}$-fiber. Let $x^{0}=\kappa, x^{1}=r, x^{2}=$ $\theta, x^{3}=\phi$. Buscher's rules specify the T-dual metric $(\tilde{g})$ and $B$-field $(\tilde{b})$ in terms of the original metric $(g)$ and $B$-field $(b)$. By Ref. [2], we have

$$
\begin{gathered}
\tilde{g}_{00}=1 / g_{00}, \tilde{g}_{0 \alpha}=b_{0 \alpha} / g_{00}, \\
\tilde{g}_{\alpha \beta}=g_{\alpha \beta}-\left(g_{0 \alpha} g_{0 \beta}-b_{0 \alpha} b_{0 \beta}\right) / g_{00} .
\end{gathered}
$$

Now, we have $b_{\alpha \beta}=0, g_{00}=H, g_{0 \alpha}=0$ by equation (2.2) above. Therefore $\tilde{g}_{00}=H, \tilde{g}_{0 \alpha}=0 \forall \alpha$. Also by equation (2.3), $\tilde{g}_{\alpha \beta}=g_{\alpha \beta}$ if $\alpha \neq 3$ or $\beta \neq 3$. We also have

$$
\begin{aligned}
\tilde{g}_{33} & =g_{33}-\left(g_{03}\right)^{2} /\left(H^{-1}\right) \\
& =H r^{2}+H^{-1}(1-\cos (\theta))^{2}-H^{-1}(1-\cos (\theta))^{2}=H r^{2} .
\end{aligned}
$$

Hence,

$$
\begin{aligned}
g_{H} & =H(d \kappa)^{2}+H(d r)^{2}+H r^{2} \sin ^{2}(\theta)(d \phi)^{2}+H r^{2}(d \theta)^{2} \\
& =H\left((d \kappa)^{2}+d \vec{r} \cdot d \vec{r}\right) .
\end{aligned}
$$

It is clear that $\tilde{g}$ is conformally equivalent to a product metric on $\mathbb{R}^{3} \times S^{1}$. As $r \rightarrow 0, H \rightarrow \infty$ thus implying that the $S^{1}$-fiber over $0 \in \mathbb{R}^{3}$ is infinitely far away from the rest of the space. This is termed as a smeared $H$-monopole solution. Quantum effects [11] are supposed to modify $H$ so that $\lim _{r \rightarrow 0} H(r, \theta)$ is finite except at the value of $\theta$ corresponding to the location of the $H$-monopole.

2) The T-dual of a set of $p$ distinct, non-intersecting NS5-branes is a $p$-center KK-monopole (see Ref. [8]). This is obtained from the previous example in the obvious fashion: we introduce $p$ sources of $H$-flux in spacetime for the $p$ NS5-branes. In the T-dual, we allow the $S^{1}$-fiber to degenerate to a point over $p$ points in the base.

Let $\left(X_{p}, g_{p}\right)$ be the spacetime containing $p$ KK-monopoles. ${ }^{10}$ This space posseses an isometric action of $S^{1}$ which is free except for $p$ fixed points. The quotient of $X_{p}$ by the $S^{1}$-action is $\mathbb{R}^{3}$ with the Euclidean metric. Let $\pi: X_{p} \rightarrow \mathbb{R}^{3}$ be the quotient map. If we use polar coordinates $(r, \theta, \phi)$ on the base $\mathbb{R}^{3}$, then we may write the metric

\footnotetext{
${ }^{10}$ This is termed a multi-Taub-NUT space.
} 
$g_{p}$ in terms of these three coordinates and an additional coordinate $\kappa$ on the $S^{1}$-fiber. Let

$$
H(\mathbf{r})=g^{-2}+\sum_{i=1}^{p} \frac{1}{2\left|\mathbf{r}-\mathbf{r}_{i}\right|},
$$

then,

$$
g_{p}=H(\mathbf{r}) d \vec{r} \cdot d \vec{r}+H(\mathbf{r})^{-1}\left(d \kappa+\frac{1}{2} \vec{\omega} \cdot d \vec{r}\right)^{2},
$$

$\nabla H=\nabla \times \vec{\omega}$. The above expression for $g_{p}$ agrees with the expression for $g_{\mathrm{TN}}$ above (see equation (2.1)) except that the expression for $H$ is different in the two cases. Since the expression for $H$ does not appear in the application of Buscher's rules above, the form of the metric on the T-dual is the same in both cases. Therefore, the metric on the T-dual of $g_{p}$ is

$$
\tilde{g}=H\left((d \kappa)^{2}+d \vec{r} \cdot d \vec{r}\right) .
$$

This is conformally equivalent to a product metric on $\mathbb{R}^{3} \times S^{1}$. The fibers over $r_{i}, i=1, \ldots, p$ are infinitely far away from the rest of the spacetime, similar to the T-dual of one KK-monopole.

Now, consider the case $p=2$. Let $Y$ be a line segment joining the image of the two centers in $\mathbb{R}^{3}$. We have $\mathbb{R}^{3}-Y \simeq S^{2} \times(0, \infty)$. Let $W=\pi^{-1}(Y)$ and consider $X_{2}-W$. Now, $\forall t, \pi^{-1}\left(S^{2} \times\{t\}\right)$ is homeomorphic to $S^{3} / \mathbb{Z}_{2}$ since each $S^{2}$ encloses $Y$ in $\mathbb{R}^{3}$. Therefore, ${ }^{11} X_{2}-$ $W \simeq\left(S^{3} / \mathbb{Z}_{2}\right) \times(0, \infty)$ (this may also be seen by examining the expression for $g_{2}$ ). Suppose we collapse $Y$ to a point in $X_{2}$ and $\pi(Y)$ to a point in $\mathbb{R}^{3}$, we would obtain an equivariant fibration $\tilde{\pi}: C\left(S^{3} / \mathbb{Z}_{2}\right) \rightarrow$ $C\left(S^{2}\right)$. Note that both $C\left(S^{3} / \mathbb{Z}_{2}\right) \simeq X_{2} / W$ and $C\left(S^{2}\right) \simeq \mathbb{R}^{3} / W$ are contractible to their vertices. This implies that $X_{2}$ is homotopy equivalent to $W=\pi^{-1}(Y) \simeq S^{2}$ (in fact equivariantly so).

If $p>2$, we may always change the total space by a homeomorphism so that the image of the $p$ centers in $\mathbb{R}^{3}$ under $\pi$ lie on a straight line $W$. The inverse image of $W$ under $\pi$ is a collection of $(p-1)$ spheres joined to each other at one point and is homeomorphic to a wedge of $(p-1)$ spheres. By an exactly similar argument to the above, $X_{p}$ is homotopy equivalent to this wedge of $(p-1)$ spheres.

3) The T-dual of a $H$-monopole of charge $p$ is a KK-monopole of charge $p .^{12}$ A $H$-monopole of charge $p$ is a fibration of the form $C^{0} S^{2} \times S^{1} \stackrel{\pi}{\rightarrow}$ $C^{0} S^{2}$. The $H$-monopole sits at some point in $\pi^{-1}(0)$ and emits $p$ units of $H$-flux on $C^{0} S^{2}-\pi^{-1}(0)$. We represent the $H$-flux as the

\footnotetext{
${ }^{11}$ i.e., the map $\pi$ restricted to any $\pi^{-1}\left(S^{2}\right) \subset\left(X_{2}-W\right)$ will be the projection map of the $S^{1}$-bundle over $S^{2}$ of Chern class 2 .

${ }^{12} \mathrm{~A}$ space with a KK-monopole of charge $p>1$ is not a smooth manifold, it possesses a conical singularity at the location of the monopole.
} 
cohomology class $[p] \in H^{3}\left(\mathrm{CS}^{2}-\{0\} \times S^{1}\right) \simeq H^{3}\left(S^{2} \times S^{1}\right)$. The KKmonopole of charge $p$ is similar to the KK-monopole configuration above, except that the fiber over each $S^{2} \times\{t\}$ in the base is a $S^{1}$ bundle of Chern class $p$, i.e., we have a fibration like $C L(1, p) \rightarrow C S^{2}$ where $L(1, p) \rightarrow S^{2}$ is the lens space viewed as a principal $S^{1}$-bundle. ${ }^{13}$

\subsection{Test Example}

To the test example, we will associate $\mathcal{A} \simeq \mathrm{CT}\left(C^{0} S^{3}, 0\right)$, as there is no $H$ flux on $\mathbb{C}^{2}$. By Ref. [6], the $\mathbb{R}$-action on $X \simeq C^{0} S^{3}$ lifts to a unique $\mathbb{R}$-action $\alpha$ on $\mathcal{A}$. We work with the example of Section 2. Suppose we consider $\mathrm{CT}(X, 0)$, then, as shown in [6], the $S^{1}$-action on $X$ lifts uniquely to a $\mathbb{R}$-action on $X$. By Theorem 4.8 of Ref. [6], the spectrum of the crossed product is homeomorphic to $(X \times \hat{\mathbb{R}}) / \sim$, where $\sim$ is the equivalence relation given by

$$
(x, \gamma) \sim(y, \chi) \Leftrightarrow \overline{\mathbb{R} \cdot x}=\overline{\mathbb{R} \cdot y}
$$

and $\gamma \bar{\chi} \in\left(\operatorname{Stab}_{x}\right)^{\perp}$. For $\mathbb{R}$, all irreps are one-dimensional, and of the form $\pi_{k}: x \mapsto \mathrm{e}^{\mathrm{i} k x}$. Distinct values of $k$ correspond to non-unitarily-equivalent irreps. (As a topological space $\mathbb{R}^{n}$ is homeomorphic to $\mathbb{R}$.) If $\gamma=k_{1}$ and $\chi=k_{2}$, then $\gamma \bar{\chi}$ corresponds to $x \mapsto \mathrm{e}^{\mathrm{i}\left(k_{1}-k_{2}\right) x}$. We have $\gamma \bar{\chi} \in\left(\operatorname{Stab}_{x}\right)^{\perp}$ iff $\gamma \bar{\chi}(l)=1, \forall l \in\left(\operatorname{Stab}_{x}\right)$. If $x \neq 0$, the stabilizer is $\mathbb{Z}$, so, we have $\mathrm{e}^{\mathrm{i}\left(k_{1}-k_{2}\right) n}=$ $1, \forall n \in \mathbb{Z}$. This implies that $\left(k_{1}-k_{2}\right)=2 l \pi, \forall l \in \mathbb{Z}$. Thus, points in $\hat{\mathbb{R}}$ are periodically identified. Further, points in the same $S^{1}$-orbit are identified. So, for $x \neq 0$, we have the dual principal bundle $S^{2} \times S^{1}$ as described in Ref. [3]. If $x=0$, the stabilizer is $\mathbb{R}$, so, we have $\mathrm{e}^{\mathrm{i}\left(k_{1}-k_{2}\right) x}=1, \forall x \in \mathbb{R}$. This implies that $k_{1}-k_{2}=0$. Thus, at the fixed point there is no quotienting.

Pick a $S^{1}$-invariant neighborhood $U$ of $0 \in \mathbb{C}^{2}$, and an $\epsilon$-neighborhood $V_{i}^{\epsilon}$ of $k_{i}$. Then, $W_{i}^{\epsilon}=U \times V_{i}^{\epsilon}$ is a neighborhood of $\{0\} \times k_{i}$ in $X \times \hat{\mathbb{R}}$. The $W_{i}^{\epsilon}$ form a neighborhood base $k_{i}$ in $X \times \hat{\mathbb{R}}$. Note that the quotient map associated to $\sim$ is open. The saturation of $W_{i}^{\epsilon}$ with respect to $\sim$ is

$$
\tilde{W}_{i}^{\epsilon}=U \times\left(\coprod_{j} V_{i}+2 \pi j\right)
$$

Thus, if $k_{i}-k_{j} \neq 2 \pi l, l \in \mathbb{Z}, \tilde{W}_{i}$ can be chosen to be disjoint from $\tilde{W}_{j}$ by tak$\operatorname{ing} V_{i}$ small enough. Conversely, if $k_{i}-k_{j}=2 \pi l$, it is impossible to choose disjoint neighborhoods for them in $X \times \hat{\mathbb{R}}$ (since the $\tilde{W}_{i}$ form a neighborhood basis at $k_{i}$ ).

\footnotetext{
${ }^{13}$ Note that $\mathrm{CL}(1, p) \simeq \mathbb{C}^{2} / \mathbb{Z}_{p}$, where $\mathbb{Z}_{p} \subseteq \mathrm{SU}(2)$ in its fundamental representation.
} 
So, we see that the crossed product has a very non-Hausdorff spectrum. In particular, its spectrum is $S^{2} \times S^{1} \times(0, \infty)$ with the line $\hat{\mathbb{R}}$ glued on at 0 . The gluing is such that if a sequence $\left\{x_{i}\right\} \in S^{2} \times S^{1} \times(0, \infty)$ converges to $x_{\infty} \in(0 \times \hat{\mathbb{R}})$, then it converges to $x_{\infty}+2 \pi l, \forall l \in \hat{\mathbb{R}}$. Note that if we remove the fixed point of the $\mathbb{R}$-action on $X$, the crossed product is a non-trivial continuous-trace algebra $\mathrm{CT}\left(S^{2} \times S^{1} \times(0, \infty), \delta^{\#}\right)$.

The crossed product $\mathcal{A} \underset{\alpha}{\rtimes} \mathbb{R}$ is not continuous trace, since $\hat{\mathcal{A}}$ is not $T_{2}$. However, it may be viewed as a $C^{*}$-bundle over $C^{0} S^{2} \times S^{1}$. Restricted to $S^{2} \times S^{1} \times(0, \infty)$, this bundle is a continuous-trace algebra with DixmierDouady invariant $\delta^{\#}$. Over $\pi^{-1}(0)$, the fiber is $C^{*}(\mathbb{R}) \otimes \mathcal{K}$ viewed as a $C^{*}$-bundle over $S^{1}$. The algebra is given by an extension of the form

$$
0 \longrightarrow \mathrm{CT}\left(S^{2} \times S^{1} \times(0, \infty), \delta^{\#}\right) \longrightarrow \mathcal{A} \rtimes \mathbb{R} \longrightarrow \mathcal{C}\left(S^{1}\right) \longrightarrow 0
$$

where $\mathcal{C}\left(S^{1}\right)$ is $C_{0}(\mathbb{R}, \mathcal{K})$ viewed as a $C^{*}$-bundle over $S^{1}$ via the quotient map $\mathbb{R} \rightarrow S^{1}$. This example can be viewed as calculating the spectrum of the group $C^{*}$-algebra of the motion group $\mathbb{C}^{2} \rtimes \mathbb{R}$.

If we view the crossed product as a $C^{*}$-bundle over the maximal Hausdorff quotient of its spectrum, we obtain a $C^{*}$-bundle over $C S^{2} \times S^{1}$. Note that $C S^{2} \times S^{1}$ is the physical T-dual. Thus, we define the physical spacetime to be the maximal Hausdorff regularization of the spectrum of the crossed product if this spectrum is non-Hausdorff.

Most physical examples of T-duality are built up from the T-duality of a NS5-brane with a KK-monopole.

Following Ref. [3], a topological space $X$ with $H$-flux $\delta$ may be naturally associated to the continuous-trace algebra $\mathrm{CT}(X, \delta)$. This $H$-flux is sourceless, i.e., we can pick a 3 -form which represents this $H$-flux in a neighborhood of every point of $X$. However, if the space possesses a source of $H$-flux, we cannot pick such a 3 -form in any neighborhood of the source. (It might be helpful to keep in mind the description of the Dirac monopole in electromagnetism: recall that the flux is not defined at the location of the monopole.) Here, the flux is only defined on $X-Y$ and so we only have a cohomology class $\delta \in H^{3}(X-Y, \mathbb{Z})$. If we could find a natural definition of a $C^{*}$-algebra $\mathcal{A}$ which encodes the structure of a space with a source of $H$ flux, then we hope that the spectrum of its crossed product with $\mathbb{R}^{n}$ would still give the T-dual. Note also that in all the physical examples above, we are dualizing spaces $X$ with a non-free $S^{1}$ action (with $X / S^{1} \simeq B$ ) and no $H$-flux, to spaces which are trivial $S^{1}$-bundles over $B$ but contain a source of $H$-flux. 
Assume that we are in the set-up of Section 2, with a space $B \times S^{1}$ with a source of $H$-flux represented by a cohomology class $\delta^{\#}$ in $H^{3}((B-$ $\left.F) \times S^{1}, \mathbb{Z}\right)$. We assume that the source is located somewhere over $F$ on $F \times S^{1} \subset B \times S^{1}$. We will replace continuous-trace algebra $\operatorname{CT}\left(B \times S^{1}, \delta^{\#}\right)$ by another $C^{*}$-algebra $\mathcal{B}$ so that the maximal Hausdorff regularisation of $\hat{\mathcal{B}}$ is $B \times S^{1}$ and we can naturally obtain a $H$-flux in $(B-F) \times S^{1}$. We suppose that $\mathcal{B}$ is an extension of the form

$$
0 \longrightarrow \mathrm{CT}\left((B-F) \times S^{1}, \delta^{\#}\right) \longrightarrow \mathcal{B} \longrightarrow \mathcal{C}(F) \longrightarrow 0,
$$

where $\mathcal{C}(F) \simeq C_{0}(\mathbb{R}) \otimes C_{0}(F) \otimes \mathcal{K}$. (Here, $\mathcal{K}$ is the $C^{*}$-algebra of compact operators on a separable infinite dimensional Hilbert space.)

For most spaces, there are many extensions $\mathcal{B}$ in equation (2.7) with the regularisation of $\hat{\mathcal{B}}$ equal to $X$ [13]. However, in this case, we can see that $\mathcal{B}$ is uniquely determined.

Lemma 2.1. Suppose we are given the following data

- topological spaces $B, F$ such that $F \subseteq B$,

- a cohomology class $\delta^{\#} \in H^{3}\left((B-F) \times S^{1}, \mathbb{Z}\right)$.

Then, topological T-duality for principal $S^{1}$-bundles with $H$-flux uniquely determines the algebra $\mathcal{B}$ in equation (2.7).

Proof. Topological T-duality applied to the trivial principal $S^{1}$-bundle $(B-F) \times S^{1} \rightarrow(B-F)$ with $H$-flux $\delta^{\#}$ gives a principal $S^{1}$-bundle $E$ over $B-F$. The characteristic class of this bundle may be obtained by integrating $\delta^{\#}$ over the $S^{1}$-fiber.

This completely determines the T-dual space $X$ up to equivariant homomorphism as follows. Any orbit of a semi-free $S^{1}$-action on a space $X$ can only have two stabilizers namely, the identity and $S^{1}$. As a result, the spaces $X$ can only have two orbit types: fixed points and free orbits. Hence, if $F$ is the subset of $B$ such that $\pi^{-1}(F)$ is the fixed point subset of $X$, by the classification theorem for spaces with two orbit types (see Ref. [7] Chapter V Section 5), the space $X$ is completely specified up to equivariant homomorphism by $F \subset B$ and the class of the principal $S^{1}$-bundle over $B-F$.

Let $\mathcal{A}=C_{0}(X, \mathcal{K})$. The $S^{1}$-action on $X$ may always be lifted to $\mathcal{A}$ uniquely, and so determines a $C^{*}$-dynamical system $(\mathcal{A}, \alpha)$ up to exterior equivalence. Now, $\mathcal{B}$ may be defined as $\mathcal{A} \underset{\alpha}{\rtimes} \mathbb{R}$. The result is unique up to $\mathrm{C}^{*}$-algebra isomorphism. 
Note that topological T-duality is a geometric operation on CW complexes by Ref. $[3,14]$ and hence may be freely used in computations. It is clear that $\mathcal{B}$ has a $\hat{\mathbb{R}}$-action $\beta$ and the crossed product $\mathcal{B} \underset{\beta}{\rtimes} \hat{\mathbb{R}}$ is isomorphic to $\mathcal{A}$.

In general, the spectrum of $\mathcal{B}$ may not be a $\mathrm{CW}$ complex. ${ }^{14}$ We emphasize that this does not imply that the physical spacetime is non-Hausdorff, only that for calculational purposes, it is convenient to take a non-Hausdorff space whose regularization is the physical spacetime.

Hence, we make the following dictionary

- Spacetime a principal $S^{1}$-bundle $X \rightarrow B$ with a sourceless $H$-flux $\delta \Leftrightarrow$ $(\mathrm{CT}(X, \delta), \alpha)$, where $\alpha$ is the lift of the $S^{1}$-action on $X$ to $\mathrm{CT}(X, \delta)$, as in Ref. [3].

- Space $B \times S^{1}$ with a NS5-brane of charge $\delta^{\#}$ wrapped on $F \times S^{1} \subseteq$ $B \times S^{1}$. $\Leftrightarrow$ The unique extension like equation (2.7) above.

If the NS5-brane is not wrapped around a $S^{1}$-orbit, for consistency, we should associate to the space an extension like equation (2.7) above. However, it is not clear how unique such an extension is. We do not address this question here, since we are T-dualizing semi-free $S^{1}$-spaces and the NS5-branes we encounter will always be wrapped around a $S^{1}$-orbit.

\section{Dyonic coordinates and KK-monpoles}

\subsection{Physical background}

It might be objected that the result obtained in the previous section is accidental. To give further evidence that it is non-trivial, we reproduce the dyonic coordinate of Refs. [11, 12, 15] within the current formalism.

In Section 1, we noted that the T-dual of a Kaluza-Klein monopole is a source of $H$-flux. ${ }^{15}$ If we apply Buscher's rules [11] to a KK-monopole we obtain a $H$-monopole smeared over the $S^{1}$-fiber over $\{0\}$. Quantum corrections are expected to localize the $H$-monopole to a particular point in the fiber. Recall that we identified a Kaluza-Klein monopole with the space $\left(\mathbb{R}^{4}, g_{\mathrm{TN}}\right)$ (termed "Taub-NUT" space). This space possesses an isometric action of $S^{1}$ with one fixed point and which is $S^{1}$-equivariantly homeomorphic to $\mathbb{C}^{2}$ with the $S^{1}$-action $\lambda \cdot\left(z_{1}, z_{2}\right)=\left(\lambda \cdot z_{1}, \lambda \cdot z_{2}\right), \lambda \in S^{1} \subseteq$ $\mathbb{C}, z_{i} \in \mathbb{C}$. We have $\mathbb{R}^{4} / S^{1} \simeq \mathbb{R}^{3}$.

\footnotetext{
${ }^{14}$ We saw this for the test example above.

${ }^{15}$ Also termed a $H$-monopole.
} 
Suppose we have a KK-monopole located somewhere in $\mathbb{R}^{4}$. By the previous section, this space is a semi-free $S^{1}$-space. As argued in the proof of Lemma 2.1, a semi-free $S^{1}$-space $X$ is completely specified by the fixed point set $F \subset B=X / S^{1}$ and the class of the principal $S^{1}$-bundle over $B-F$. Thus, it is specified by a class $\lambda$ in $H^{2}(B-F)$.

Here, it would be specified by the class of the principal $S^{1}$-bundle $\mathbb{R}^{4}-\mathbf{x} \rightarrow \mathbb{R}^{3}-\mathbf{x}$, and the fixed point set $\mathbf{x} \subseteq \mathbb{R}^{3}$. For the case of a single KK-monopole, the isomorphism class of the principal $S^{1}$-bundle is fixed. ${ }^{16}$ Thus, we only need to know $\mathbf{x}$ to fix the KK-monopole space. Hence only three numbers are needed to specify the KK-monopole, ${ }^{17}$ namely the location of the image of the center in $\mathbb{R}^{3}$ under the quotient map. In the T-dual picture, we have a source of $H$-flux somewhere in the fiber over $\{0\} \times S^{1}$. This is specified by the position of the source in $\mathbb{R}^{3}$ and the location of the source in $\{0\} \times S^{1}$. Thus four parameters are needed to specify the Tdual. Since T-dual spaces are physically equivalent, we should need the same number of parameters on both sides. It is interesting, therefore, to ask which datum of the KK-monopole changes when we change the location of the source of $H$-flux in the $S^{1}$-fiber of the T-dual. According to a result of Sen [15] (see also [11, 12]), this may be obtained as follows. On the total space of the KK-monopole, we have a zero $H$-flux. This implies that the gauge field $B$ of the $H$-flux is a closed 2-form (as $H=d B$ ). Since $\mathbb{R}^{4}$ is contractible, $B$ is also exact. However, for the multi-Taub-NUT metrics, there exist ${ }^{18} L^{2}$-normalized 2-forms $\Omega_{i}$ such that $\Omega_{i} \not \rightarrow 0$ as $|\mathbf{x}| \rightarrow \infty$. It is scalar multiples of these forms that give rise to non-trivial $B$-fields. It is this $B$-field that corresponds to the position of the $H$-monopole in the T-dual. It is termed a "dyonic coordinate" in Refs. $[11,12]$ by analogy with the case of monopoles in gauge theories [17]. If the $B$-field changes in time according to $B(t)=\beta(t) \Omega$ then on the T-dual side, (see below) this corresponds to changing the angular coordinate of the $S^{1}$ factor of $\mathbb{R}^{3} \times S^{1}$ via an isometry

$$
\kappa(t)=\kappa(0)-\beta(t) .
$$

We may explicitly calculate ${ }^{19}$ the above effect using equation (2.3). Recall, that the Taub-NUT metric was

$$
\begin{aligned}
g_{\mathrm{TN}}(r, \theta, \phi, \kappa)= & H d \vec{r} \cdot d \vec{r}+H^{-1}\left(d \kappa^{2}+(1-\cos (\theta)) d \phi d \kappa\right. \\
& \left.+(1-\cos (\theta))^{2} d \phi^{2}\right) .
\end{aligned}
$$

\footnotetext{
${ }^{16}$ It must be the unique bundle over $S^{2} \times(0, \infty)$ with Chern class 1 .

${ }^{17}$ Following Ref. [11, page $\left.2-3\right]$.

${ }^{18}$ Note that the total space is noncompact, so the usual Hodge theorem does not apply here. See Ref. [16, Section 7].

${ }^{19}$ We follow Ref. [11] here.
} 
Its T-dual in the absence of a $B$-field was the $H$-monopole metric

$$
g_{H}(r, \theta, \phi, \kappa)=H(d \kappa)^{2}+H(d r)^{2}+H r^{2} \sin ^{2}(\theta)(d \phi)^{2}+H r^{2}(d \theta)^{2}
$$

Note that for the Taub-NUT metric, $g_{00}=H^{-1}$; the harmonic form $B$ discussed above is given by

$$
\begin{aligned}
B & =\beta \Omega=\beta d \Lambda=\beta d\left(\frac{1}{g^{2} H}\left(d \kappa+\frac{(1-\cos (\theta))}{2} d \phi\right)\right) \\
& =-\frac{\beta H^{\prime}}{g^{2} H^{2}} d r\left(d \kappa+\frac{(1-\cos (\theta))}{2} d \phi\right)-\frac{\beta}{g^{2} H} \sin \theta d \theta d \phi
\end{aligned}
$$

Hence we have $b_{01}=-\frac{\left(\beta H^{\prime}\right)}{\left(g^{2} H^{2}\right)}$ and so

$$
\tilde{g}_{00}=H, \tilde{g}_{01}=-\frac{\left(\beta H H^{\prime}\right)}{\left(g^{2} H^{2}\right)}=-\frac{\left(\beta H^{\prime}\right)}{\left(g^{2} H\right)} .
$$

Thus, the T-dual is given by

$$
\begin{aligned}
\tilde{g} & =H(r)\left(d \kappa^{2}-\frac{\left(\beta H^{\prime}\right)}{\left(g^{2} H\right)} d \kappa d r\right)+\left\{\text { terms of } g_{\alpha \beta}, \beta \neq 0, \alpha \neq 0\right\} \\
& =H(r)\left(d \kappa^{2}-\left(\beta H^{\prime}\right)\left(g^{2} H^{2}\right) d \kappa d r+\left(\beta^{2} H^{\prime 2}\right) /\left(4 g^{4} H^{4}\right) d r^{2}\right)+\cdots \\
& =H(r)\left(\left(d \kappa-\left(\beta H^{\prime}\right) /\left(g^{2} H^{2}\right) d r\right)^{2}\right)+\cdots \\
& =H(r)\left(d\left(\kappa+\beta /\left(g^{2} H\right)\right)\right)^{2}+\cdots
\end{aligned}
$$

Therefore, if we take a diffeomorphism $\Gamma$ of the T-dual, $\Gamma: \mathbb{R}^{3} \times S^{1} \rightarrow$ $\mathbb{R}^{3} \times S^{1}$ given by

$$
r=r, \theta=\theta, \phi=\phi, K=\kappa+\frac{\beta}{\left(g^{2} H(r)\right)},
$$

we see that $\tilde{g}(r, \theta, \phi, \kappa)=\Gamma^{*}\left(g_{H}(r, \theta, \phi, K)\right)$. Also, $\Gamma$ is an isometric diffeomorphism ${ }^{20}$ between the distinct Riemannian manifolds $\left(\mathbb{R}^{3} \times S^{1}, g_{H}\right)$ and $\left(\mathbb{R}^{3} \times S^{1}, \tilde{g}\right)$. Note that as $r \rightarrow \infty, \Gamma$ approaches the isometry $\kappa \rightarrow \kappa+\beta$. In general, it is preferable if such transformations approach the identity at infinity. Thus, we consider instead the diffeomorphism $\Lambda$ of the T-dual,

\footnotetext{
${ }^{20}$ Therefore, by the principle of general covariance, these two are indistinguishable at the level of general relativity, as expected.
} 
$\Lambda: \mathbb{R}^{3} \times S^{1} \rightarrow \mathbb{R}^{3} \times S^{1}$ given by

$$
r=r, \theta=\theta, \phi=\phi, \tilde{K}=\kappa+\frac{\beta}{\left(g^{2} H(r)\right)}-\beta,
$$

which approaches the identity as $r \rightarrow \infty$, we obtain that

$$
\tilde{g}(r, \theta, \phi, \kappa)=\Lambda^{*}\left(g_{H}(r, \theta, \phi, \tilde{K}+\beta)\right) .
$$

We expect that it should be possible to model the dyonic coordinate discussed above within the formalism of Refs. [3, 4]. We expect this because the above T-dual was also obtained from Buscher's rules. If we could mimic this effect within the topological formalism, this would give added evidence that the T-dual in Chapter (1) is the "correct" one.

\subsection{Mathematical formalism}

We first simplify the problem by passing to a suitable compactification. We view $\mathbb{C}^{2}$ as an open subset of $\mathbb{C P}^{2}$ : i.e., we have compactified $\mathbb{C}^{2}$ by adding an $S^{2}$ at $\infty$ obtained by collapsing each $S^{1}$-orbit to a point. As $H^{2}\left(\mathbb{C P}^{2}, \mathbb{Z}\right) \simeq \mathbb{Z}$, and $\mathbb{C P}^{2}$ is compact, there is a unique harmonic form on $\mathbb{C P}^{2}$ whose integer multiples generate $H^{2}\left(\mathbb{C P}^{2}, \mathbb{Z}\right) \subseteq H^{2}\left(\mathbb{C P}^{2}, \mathbb{R}\right)$. It is shown in Ref. [16] that the restriction of this form to $\mathbb{C}^{2} \subseteq \mathbb{C P}^{2}$ is related to $\Omega$. Also $\beta(t) \Omega$ extends to a closed distributional 2-form on $\mathbb{C P}^{2}$ and gives rise [16] to an element of $H^{2}\left(\mathbb{C P}^{2}, \mathbb{R}\right)$. It is not clear which topological object may be associated with a real cohomology class. However, if we restrict ourselves to integral $B$-fields (i.e., elements of the form $m \Omega, m \in \mathbb{Z}$ ) we may reformulate the above as follows. If we change the $B$-field by adding an element of $H^{2}\left(\mathbb{C P}^{2}, \mathbb{Z}\right)$ to it, on the T-dual side, this should correspond to rotating the $S^{1}$-fiber via equation (3.1).

We use homogenous coordinates $\left[x_{1}: x_{2}: x_{3}\right]$ on $\mathbb{C P}^{2}$ (with $x_{i} \in \mathbb{C}$ and $\left.\left(x_{1}: x_{2}: x_{3}\right) \sim\left(\lambda x_{1}: \lambda x_{2}: \lambda x_{3}\right), \forall \lambda \in \mathbb{C}^{*}\right)$ Then $\mathbb{C}^{2}$ corresponds to the subset

$$
U=\left\{\left[x_{1}: x_{2}: x_{3}\right] \mid x_{3} \neq 0\right\}
$$

and the sphere at infinity to the subset

$$
W=\left\{\left[x_{1}: x_{2}: x_{3}\right] \mid x_{3}=0\right\} .
$$

We consider the action $\lambda \cdot\left[x_{1}: x_{2}: x_{3}\right]=\left[\lambda x_{1}: \lambda x_{2}: x_{3}\right], \lambda \in S^{1}$ on $\mathbb{C P}^{2}$. This is obviously well defined. Note that on $U$ it turns into the action of Section 2 
because the following commutes

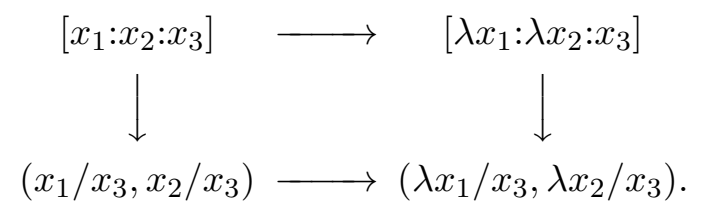

Hence we have an $S^{1}$-action on $\mathbb{C P}^{2}$ which we may lift to a $\mathbb{R}$-action $\alpha_{t}$ on $C\left(\mathbb{C P}^{2}, \mathcal{K}\right)$. Any 2 -form $\lambda \in H^{2}\left(\mathbb{C P}^{2}, \mathbb{Z}\right)$ gives rise to a spectrum-fixing automorphism of $C\left(\mathbb{C P}^{2}, \mathcal{K}\right)$. We recall that spectrum-fixing automorphisms of $C^{*}$-algebras are classified up to inner automorphisms by their PhillipsRaeburn invariant which is a homomorphism $\zeta: \operatorname{Aut}_{C_{0}(X)}(\mathcal{A}) \rightarrow H^{2}(X, \mathbb{Z})$. The following theorem shows that this automorphism may always be "dualized":

Theorem 3.1. Let $X$ be a finite $C W$ complex with a semi-free $S^{1}$-action. Let $\alpha_{t}$ be a lift of the $S^{1}-$ action on $X$ to $C(X, \mathcal{K})$.

1. Let $[\lambda] \in H^{2}(X, \mathbb{Z})$. Then, there is a $\mathbb{R}$-action $\beta$ on $C(X, \mathcal{K})$ exterior equivalent to $\alpha$ and a spectrum-fixing $\mathbb{Z}$-action $\lambda$ on $C(X, \mathcal{K})$ which has Philips-Raeburn obstruction $[\lambda]$ such that $\beta$ and $\lambda$ commute.

2. With the notation above, the action $\lambda$ induces a $\mathbb{Z}$-action $\tilde{\lambda}$ on $C(X, \mathcal{K})$ $\rtimes \mathbb{R}$. The induced action on the crossed product is locally unitary on the spectrum of the crossed product and is thus spectrum fixing.

Proof. Let $A=C(X, \mathcal{K})$ then $X=\hat{A}$.

1. We have a short exact sequence

$$
0 \longrightarrow \operatorname{Inn}(A) \longrightarrow \operatorname{Aut}_{C_{0}(X)}(A) \stackrel{\zeta}{\longrightarrow} H^{2}(X, \mathbb{Z}) \longrightarrow 0 .
$$

Pick any $\mathbb{Z}$-action $\tilde{\lambda}$ with $\zeta(\tilde{\lambda})=[\lambda]$. Note that $\zeta\left(\alpha_{-t} \tilde{\lambda} \alpha_{t}\right)=\alpha_{t}^{*}(\zeta(\lambda))$ (By Lemma 4.4 of Ref. [18]). Since $\alpha_{t}^{*}=i d, \forall t \in \mathbb{R}\left(\right.$ as $\left.\alpha_{1}^{*}=i d\right)$, we have $\zeta\left(\alpha_{-t} \tilde{\lambda} \alpha_{t}\right)=[\lambda], \forall t \in \mathbb{R}$. We see that $\alpha_{-t} \lambda \alpha_{t}$ is equal to $\lambda$ up to inner automorphisms. If it were exactly equal to $\lambda$ we would obtain a map $\phi: \mathbb{R} \times \mathbb{Z} \rightarrow \operatorname{Aut}(A)$. As it is, we obtain a map $\tilde{\phi}: \mathbb{R} \times \mathbb{Z} \rightarrow$ $\operatorname{Out}(A)$. To lift this to $\operatorname{Aut}(A)$, by Ref. [18], Lemma 4.6, an obstruction class in $H_{M}^{3}(\mathbb{R} \times \mathbb{Z}, C(X, \mathbb{T}))$ must vanish. To calculate this cohomology group, we use the fact that $H_{M}^{3}(G, A) \simeq \underline{H}_{M}^{3}(G, A)$ if $G$ is second countable and locally compact and $A$ is a polish abelian $G$-module (see [19, Theorem 7.4]). Now, by Ref. [20, Theorem 9], there is a spectral sequence ${ }^{21}$ converging to $\underline{H}^{*}(\mathbb{R} \times \mathbb{Z}, C(X, \mathbb{T}))$ whose $E^{2}$ term is $E_{p, q}^{2}=\underline{H}^{p}\left(\mathbb{Z}, \underline{H}^{q}(\mathbb{R}, C(X, \mathbb{T}))\right.$. By Ref. [6, Theorem 4.1], we have that

\footnotetext{
${ }^{21}$ See also Ref. [19, page 190].
} 
$H^{q}(\mathbb{R}, C(X, \mathbb{T}))=0, q>1$. Note that the $\mathbb{Z}$-module $H_{M}^{*}(\mathbb{R}, C(X, \mathbb{T}))$ has a trivial $\mathbb{Z}$-action, since $C(X, \mathbb{T})$ has a trivial $\mathbb{Z}$-action. Since $\mathbb{Z}$ has the discrete topology, the Borel cochains with values in a Polish abelian group $A$ with a trivial $\mathbb{Z}$-action are simply all the cochains. Therefore, $H_{M}^{*}(\mathbb{Z}, A) \simeq H^{*}(\mathbb{Z}, A)$, where the last cohomology group is calculated by the usual group cohomology theory. Since $H^{k}(\mathbb{Z}, A)=0$ for $k>1$, we see that $E_{2}^{p, q}=0$ for $p>1$ as well as for $q>1$. Thus $E_{2}^{p, q}=0$ for all $p+q=3$ and $H^{3}$ vanishes. As a result, the action $\tilde{\phi}$ lifts to a twisted action $\phi^{\prime}$. By Raeburn's Stabilization Trick, this is exterior equivalent to an ordinary action $\phi$.

Note that the restriction of $\phi$ to the $\mathbb{R}$-factor gives an $\mathbb{R}$-action exterior equivalent to $\alpha$ (since the lift of the $S^{1}$-action is unique up to exterior equivalence).

2. Note that $\lambda$ is a locally unitary action. Hence, we may pick a sufficiently small open set $U \subset X$ such that $\lambda$ is unitary on the localization $\mathcal{A}_{U}$ of $\mathcal{A}$ to $U$. This defines an element $f \in C_{b}\left(\mathbb{R}, \operatorname{UM}\left(\mathcal{A}_{U}\right)\right)$ by $f(t)=u_{\alpha}, \forall t$. Note that $U$ may be taken to be $S^{1}$-invariant. Since there are two orbit types and we're assuming everything is homotopy finite, we can choose $U$ to be equivariantly homeomorphic to either $S^{1} \times V$ with $V$ contractible (if we're away from a fixed point) or to a cone times $V, V$ a contractible open subset of the fixed set. For both these, $H^{2}(U, \mathbb{Z}) \simeq 0$, so $\lambda$ localized to these sets is unitary. Now, since $U$ is a union of $S^{1}$-orbits, $C_{b}\left(\mathbb{R}, \operatorname{UM}\left(\mathcal{A}_{U}\right)\right) \subseteq \operatorname{UM}\left(\mathcal{A}_{U} \rtimes \mathbb{R}\right)$. The induced automorphism on $\mathcal{A}_{U} \rtimes \mathbb{R}$ is given on $C_{0}\left(\mathbb{R}, \mathcal{A}_{U}\right)^{\lambda}$ (which is a dense subspace of $\left.\mathcal{A}_{U} \underset{\lambda}{\rtimes} \mathbb{R}\right)$ by $\tilde{\lambda}(g)(s)=f(s)^{*} g(s) f(s), \forall s \in \mathbb{R}$, this extends to a unitary automorphism of $\mathcal{A}_{U} \rtimes \mathbb{R}$. Thus $\tilde{\lambda}$ is locally unitary on the spectrum of the crossed product.

Thus we see that under T-duality, a class in $H^{2}\left(\mathbb{C P}^{2}, \mathbb{Z}\right)$ gives rise to a spectrum fixing automorphism of the crossed product algebra. We identify this with a rotation of the form equation (3.1) with $\beta=2 m \pi, m \in \mathbb{Z}$.

Note that in our example, the spectrum $X^{\#}$ of the crossed product is not Hausdorff. Hence, the crossed product algebra is not continuous trace. Thus, this spectrum fixing automorphism does not define a cohomology class in $H^{2}(Y, \mathbb{Z})$ where $Y$ is the Hausdorff regularization of $X^{\#}$. Physically, this is reasonable, since there is an $H$-flux in the T-dual so we would not expect a $B$-field there. 


\subsection{Multiple KK-monopoles}

Consider the Multi-Taub-NUT space $^{22} X_{k}$. Using the coordinates given there, ${ }^{23}$ the metric on $X_{k}$ is given by

$$
\begin{aligned}
g_{k \mathrm{TN}}= & H(\overrightarrow{\mathbf{r}}) d \overrightarrow{\mathbf{r}} \cdot d \overrightarrow{\mathbf{r}}+H(\overrightarrow{\mathbf{r}})^{-1}\left(d \kappa+\frac{1}{2} \omega \cdot d \overrightarrow{\mathbf{r}}\right)^{2} \\
& \text { where } H(\overrightarrow{\mathbf{r}})=1+\sum_{i=1}^{k} \frac{1}{\mid \overrightarrow{\mathbf{r}}-\overrightarrow{\mathbf{r}_{i} \mid}} .
\end{aligned}
$$

The T-dual is given by

$$
g_{H_{k}}(r, \theta, \phi, \kappa)=H\left((d \kappa)^{2}+d \vec{r} \cdot d \vec{r}\right)
$$

The harmonic forms $B_{k}$ on $X_{k}$ are given by

$$
\begin{gathered}
B_{i}=\beta \Omega_{i}=\beta d \xi_{i}, \\
\xi_{i}=\alpha_{i}-\frac{H_{i}}{H}(d \kappa+\alpha), \\
H_{i}=\frac{1}{\left|r-r_{i}\right|}, \\
B_{i}=-\beta \frac{\partial}{\partial r}\left(\frac{H_{i}}{H}\right) d \kappa d r+\{\text { terms not containing } d \kappa\}, \\
B_{01 i}=-\beta \frac{\partial}{\partial r}\left(\frac{H_{i}}{H}\right)
\end{gathered}
$$

Hence, for the T-dual metric,

$$
g_{00}=H, \quad g_{01}=-\beta \frac{\partial}{\partial r}\left(\frac{H_{i}}{H}\right)
$$

As in the previous section, the T-dual is given by

$$
g_{H_{k}}=H(r)\left(d\left(\kappa+\beta \frac{\partial}{\partial r}\left(\frac{H_{i}}{H}\right)\right)\right)^{2}+\left\{\text { terms of } g_{\alpha \beta}, \quad \alpha \neq 0, \beta \neq 0\right\} .
$$

We prefer to take the following as a basis for the set of harmonic forms

$$
\tilde{B}_{i}=\sum_{j, j \neq i} B_{i}=-\beta \frac{\partial}{\partial r}\left(1-\frac{H_{i}}{H}\right) d \kappa d r+\cdots
$$

Then, the T-dual is given by

$$
g_{H_{k}}=H(r)\left(d\left(\kappa+\beta \frac{\partial}{\partial r}\left(1-\frac{H_{i}}{H}\right)\right)\right)+\left\{\text { terms of } g_{\alpha \beta}, \quad \alpha \neq 0, \beta \neq 0\right\} .
$$

\footnotetext{
${ }^{22}$ defined in Chapter 1.

${ }^{23}$ see equation (2.5).
} 
We take the diffeomorphism $\Gamma$ of $\mathbb{R}^{3} \times S^{1}$ given by

$$
\begin{gathered}
r=r, \\
\theta=\theta, \\
\phi=\phi, \\
\kappa=\kappa+\beta \frac{\partial}{\partial r}\left(1-\frac{H_{i}}{H}\right)-\beta .
\end{gathered}
$$

Hence, exactly in the previous section, $g_{\mathrm{TN}_{k}}(r, \theta, \phi, \kappa)=\Lambda^{*}\left(g_{H_{k}}(r, \theta\right.$, $\phi, \kappa+\beta))$.

The calculation in the previous subsection trivially extends to multi-TaubNUT spaces. We need a suitable compactification $\tilde{X}_{k}$ of $X_{k}$ such that the harmonic forms on $X_{k}$ are related to the cohomology of $\tilde{X}_{k}$. Since $g_{\mathrm{TN}_{k}}$ is a metric of fibered boundary type [16], we use the compactification given in that paper. Thus, $\tilde{X}_{k}$ is obtained by collapsing the $S^{1}$-fibres of $S^{3} / \mathbb{Z}_{k}$ (which is the boundary of $X_{k}$ at $\infty$ ) to points to obtain an $S^{2}$. We find from [16] that the $L^{2}$-harmonic forms on $X_{k} \subseteq \tilde{X}_{k}$ are related the harmonic forms which are the elements of $H^{2}\left(\tilde{X}_{k}, \mathbb{Z}\right)$. Now, $X_{k}$ has an $S^{1}$-action which extends to $\tilde{X}_{k}$ by fixing the $S^{2}$ at $\infty$. In Theorem (3.1), applies to any finite CW complex $X$ which possesses an $S^{1}$-action. This would be be the analogue of the dyonic coordinate for $X_{k}$. In general, we can repeat the above construction for any Riemannian manifold of fibered boundary type using the compactification in Ref. [16].

\section{Gerbes and T-duality}

In this section, we show that we may naturally associate a 2-gerbe (see below) to a semi-free $S^{1}$-space. Similarly, we show that we may associate a 3 -gerbe to a space with a source of $H$-flux. We show that topological T-duality induces a natural map between these two gerbes.

We are trying to model a space containing a source of $H$-flux, a 3 -form field. It is useful to begin by studying a simpler example, a Dirac monopole (this is a source of a 2-form field, the electromagnetic flux). We begin by reviewing a construction of J.-L. Brylinski [21].

\subsection{The Dirac monopole}

It is well known that a Dirac monopole situated at $\mathrm{x} \in \mathbb{R}^{3}$ defines a line bundle $E$ on $\mathbb{R}^{3}-\mathrm{x}$, the gauge bundle, together with a connection $\nabla_{X}$ on 
this line bundle. The connection is only specified up to a gauge transformation, i.e., we consider $\nabla_{X}$ equivalent to $U \nabla_{X} U^{*}$, where $U$ is a section of the bundle $\operatorname{End}(E)$.

The curvature of this connection is identified with the electromagnetic field strength $F$ emitted by the monopole. It is a closed 2 -form on $\mathbb{R}^{3}-\mathrm{x}$. Following Refs. [21, 22], we view the field strength of the monopole $F$ as a distribution-valued 2-form on $\mathbb{R}^{3}$ with singular support at x. Maxwell's equations for the field generated by the monopole are $d F=l$ and $d * F=h$, where $l, h$ are distribution-valued differential forms with support at the point $\mathrm{x}$ in $\mathbb{R}^{3}$; these model the monopole as a source of the electromagnetic field.

(From now on, we assume that the monopole is located at $\mathrm{x}=0$. We will relax this restriction later.) From the equations governing $F$, it follows that $F$ diverges as $F \sim 1 /|y|^{\alpha}$ for some constant $\alpha$ as $\mathbf{y} \rightarrow 0$

Classically, a line bundle with connection has a characteristic class which is the de Rham cohomology class of its curvature 2-form. In Refs. [22, 21], the authors obtain a generalization of this characteristic class for the monopole as follows. Suppose we attempt to use the curvature of the line bundle associated to the monopole to obtain a cohomology class. This class would most naturally reside in the cohomology of the complex $\Omega_{\{0\}}^{*}\left(\mathbb{R}^{3}\right)$ which consists of distribution-valued differential forms on $\mathbb{R}^{3}$ which have singular support at $0 \in \mathbb{R}^{3}$. It can be shown that the cohomology of this complex is $H^{*}\left(\mathbb{R}^{3}, \mathbb{R}^{3}-0\right)$. Using the long exact sequence of the pair $\left(\mathbb{R}^{3}, \mathbb{R}^{3}-0\right)$, we obtain the exact sequence

$$
H^{2}\left(\mathbb{R}^{3}\right) \longrightarrow H^{2}\left(\mathbb{R}^{3}-0\right) \longrightarrow H^{3}\left(\mathbb{R}^{3}, \mathbb{R}^{3}-0\right) \longrightarrow H^{3}\left(\mathbb{R}^{3}\right)
$$

Since all the cohomology groups of $\mathbb{R}^{3}$ vanish, we obtain an isomorphism $H^{2}\left(\mathbb{R}^{3}-0\right) \rightarrow H^{3}\left(\mathbb{R}^{3}, \mathbb{R}^{3}-0\right)$ under which $\left[F \mid\left(\mathbb{R}^{3}-\{0\}\right)\right] \rightarrow[d F=$ l]. Now, as explained in Ref. [21] we would like to move the monopole about $\mathbb{R}^{3}$ without affecting the above class. This may be done by passing to $S^{3}$ (which is to be viewed as) the 1-point compactification of $\mathbb{R}^{3}$. We assume given an inclusion $i: \mathbb{R}^{3} \rightarrow S^{3}$ which induces maps $i^{*}, j^{*}$ as shown below.

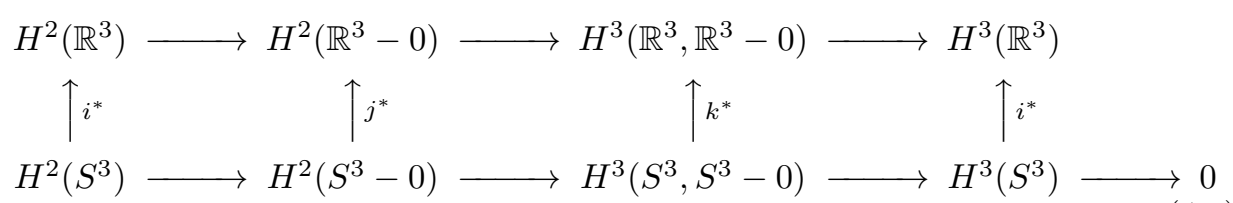


Here $k^{*}$ is an isomorphism by excision. This gives the following commutative diagram with exact rows

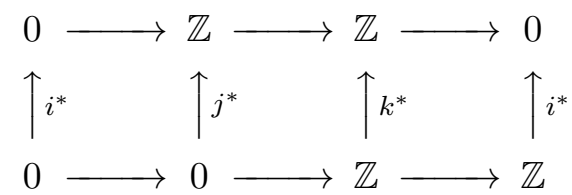

and so an isomorphism of $H^{3}\left(\mathbb{R}^{3}, \mathbb{R}^{3}-0\right)$ with $H^{3}\left(S^{3}\right)$.

Hence, given a monopole situated at 0 in $\mathbb{R}^{3}$, we view its curvature 2 -form as a distribution-valued 2-form defined on $\mathbb{R}^{3}$ with singular support at 0 . Its cohomology class gives an element of $H^{3}\left(\mathbb{R}^{3}, \mathbb{R}^{3}-0\right)$ which, by the above isomorphism, gives a class in $H^{3}\left(S^{3}\right)$. It is also clear by the above argument that changing the location of the monopole from 0 to $\mathrm{x} \in \mathbb{R}^{3}$ will not change the above class in $H^{3}\left(S^{3}\right)$.

It is interesting to view the class obtained above in a geometric way which will be useful later. Elements of $H^{3}\left(S^{3}\right)$ are in one-to-one correspondence with stable continuous-trace $C^{*}$-algebras with spectrum $S^{3}$. Given a monopole on $\mathbb{R}^{3}$, is it possible to uniquely obtain a continuous-trace algebra on $S^{3}$ ? Also, does every continuous-trace algebra on $S^{3}$ arise in this way?

Suppose we are given a monopole located at a point $\mathrm{x} \in \mathbb{R}^{3}$. This gives rise to a closed 2-form (the gauge field strength) on $\mathbb{R}^{3}-\mathrm{x}$. The de Rham cohomology class $\omega$ of this 2 -form defines an element ${ }^{24}$ in $H^{2}\left(\mathbb{R}^{3}-\mathrm{x}, \mathbb{Z}\right)$ and thus a map $\left(\mathbb{R}^{3}-\mathbf{x}\right) \rightarrow K(\mathbb{Z}, 2)$. Since $K(\mathbb{Z}, 2)$ is homotopy equivalent to $\mathcal{P U}$ we obtain a map from $\left(\mathbb{R}^{3}-\mathrm{x}\right) \rightarrow \mathcal{P U}$. (Note that $\mathbb{R}^{3}-\mathrm{x} \simeq S^{3}-\{\mathrm{x}, \infty\}$.) Using the gluing construction of continuous-trace algebras on $S^{3}$ given in Ref. [13], we see that we obtain a unique stable continuous-trace algebra on $S^{3}$ associated to the monopole. ${ }^{25}$ Every class in $H^{3}\left(S^{3}\right)$ arises in this way because the Dixmier-Douady invariant of the continuous-trace algebra, which classifies the algebra up to isomorphism, is the image of $\omega$ in $H^{3}\left(S^{3}\right)$ via the isomorphism $H^{2}\left(S^{2}\right) \rightarrow H^{3}\left(S^{3}\right)$.

Note that the same algebra on $S^{3}$ represents the gauge bundle of a monopole at any other ${ }^{26}$ point $x$ in $\mathbb{R}^{3}$. Pick as the open sets $U_{\mathrm{x}}=S^{3}-x$ and $U_{\infty}=S^{3}-\infty$. Since these open sets are contractible, the algebra localized to these sets is $C_{0}\left(U_{*}, \mathcal{K}\right)$. We obtain transition functions on $U_{\mathbf{x}} \cap U_{\infty}$ which define the same class in $H^{2}\left(S^{2}\right)$ as above, since the Dixmier-Douady

\footnotetext{
${ }^{24}$ The form is integral because of "Dirac Quantization".

${ }^{25}$ Take trivial continuous-trace algebras over $S^{3}-x$ and $S^{3}-\infty$ and glue on the overlap using the above function, noting that $\mathcal{P} U \simeq \operatorname{Aut}(\mathcal{K})$.

${ }^{26}$ Hence the associated continuous-trace algebra does not determine the monopole completely.
} 
invariant is the same. This shows that we have a line bundle defined on $U_{\mathrm{x}} \cap U_{\infty}$ which we identify with the gauge bundle of a monopole located at $\mathrm{x}$.

\subsection{Sources of $H$-flux}

For the first T-dual pair, we have a source of $H$-flux situated at $0 \times S^{1}$ on $C^{0} S^{2} \times S^{1}$. We represent such a situation by a distribution-valued 3 -form on $C^{0} S^{2} \times S^{1}$ with singular support at $0 \times S^{1}$. As above, we expect that its cohomology class in a suitably defined group should give a topological invariant of this situation. By an argument similar to the one given above, the cohomology class should lie in $H^{4}\left(C^{0} S^{2} \times S^{1}, 0 \times S^{1}\right)$. We have an exact sequence,

$$
\begin{aligned}
H^{3}\left(C^{0} S^{2} \times S^{1}\right) \rightarrow & H^{3}\left(\left(C^{0} S^{2}-0\right) \times S^{1}\right) \\
\phi^{*} & \\
& H^{4}\left(C^{0} S^{2} \times S^{1},\left(C^{0} S^{2}-0\right) \times S^{1}\right) \rightarrow H^{4}\left(C^{0} S^{2} \times S^{1}\right)
\end{aligned}
$$

As $C^{0} S^{2} \times S^{1}$ is homotopy equivalent to $S^{1}$, we get an isomorphism $\left.\phi^{*}: H^{3}\left(\left(C^{0} S^{2}-0\right) \times S^{1}\right) \rightarrow H^{4}\left(C^{0} S^{2} \times S^{1},\left(C^{0} S^{2}-0\right) \times S^{1}\right)\right)$. Now consider the inclusion $C^{0} S^{2} \times S^{1} \rightarrow S^{3} \times S^{1}$, where we view $S^{3}$ as the 1-point compactification of $C^{0} S^{2}$. We get a commutative diagram

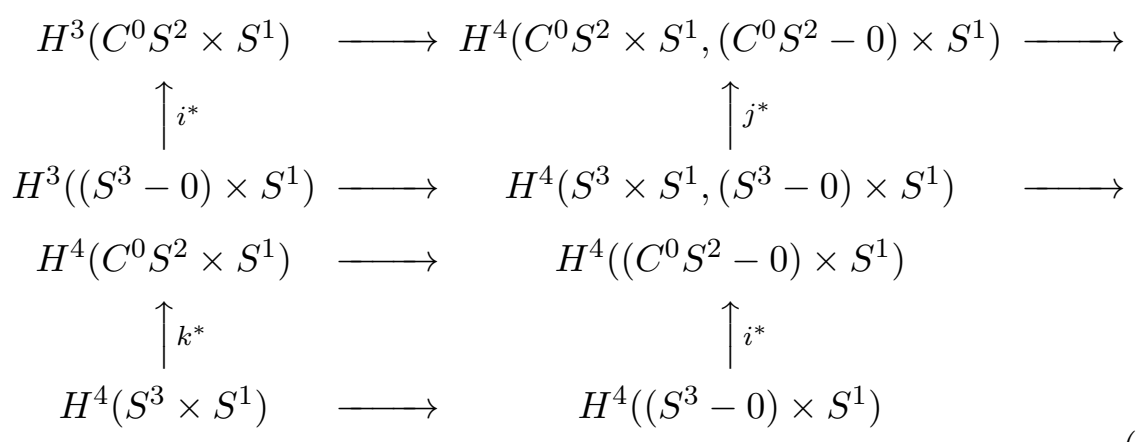

and hence an isomorphism $H^{4}\left(C^{0} S^{2} \times S^{1},\left(C^{0} S^{2}-0\right) \times S^{1}\right) \simeq H^{4}\left(S^{3} \times S^{1}\right)$.

We would like a geometric version of this isomorphism; i.e., given an extension like equation (2.6), we would like to naturally associate a class in $H^{4}\left(S^{3} \times S^{1}\right)$. We use the above argument to associate a 2 -gerbe (see below) on $B^{+}$to a semi-free $S^{1}$-space $X$ with $X / S^{1}=B$. We show in Theorem. (4.3) below that T-duality gives a natural mapping between 2-gerbes on $B$ and 3-gerbes on $B^{+} \times S^{1}$. The characteristic class of this 3 -gerbe is exactly the class obtained above. 


\subsection{Gerbes}

Following Ref. [23], we define a $k$-gerbe on a space to be a geometric object whose isomorphism classes are naturally associated to a class in $H^{k}\left(X ; \underline{\mathbb{C}^{*}}\right) \simeq$ $H^{k+1}(X ; \mathbb{Z})$ where $\mathbb{C}^{*}$ is the sheaf of germs of $\mathbb{C}^{*}$-valued functions on $X$. Therefore, ${ }^{27}$ a 0 -gerbe is just an element of $C\left(X, \mathbb{C}^{*}\right)$ up to homotopy. A 1-gerbe is a line bundle (since isomorphism classes of such objects are in one-to-one correspondence with elements of $\left.H^{1}\left(X ; \mathbb{C}^{*}\right)\right)$. A 2-gerbe is an object whose isomorphism classes are naturally associated to an element of $H^{2}\left(X ; \mathbb{C}^{*}\right) \simeq H^{3}(X ; \mathbb{Z})$ and may be identified with a continuous-trace algebra on $X$ up to isomorphism. No explicit realizations of gerbes above degree 2 are known, but they may be easily specified (see Ref. [24] Section 4.5) in terms of data similar to that given below for 2-gerbes.

Definition 4.1. A abelian, locally-trivialized 2 -gerbe ${ }^{28}$ on a space $X$ is specified by the following data

- An open cover of $X$

$$
\left\{U_{i}: i \in I\right\} \quad \text { with } \bigcup_{I} U_{i}=X
$$

(and we write $U_{i, j}=U_{i} \cap U_{j}$ and so on for other tuples of indices).

- A complex line-bundle $\Gamma_{j}^{i}$ over $U_{i, j}$ for each ordered pair $(i, j), i \neq j$, such that $\Gamma_{i}^{j}$ and $\Gamma_{j}^{i}$ are dual to each other.

- For each ordered triple of distinct indices $(i, j, k)$, a nowhere-zero section

$$
\theta_{i, j, k} \in \Gamma\left(U_{i, j, k} ; \Gamma_{i}^{j} \otimes \Gamma_{j}^{k} \otimes \Gamma_{k}^{i}\right)
$$

such that the sections $\theta_{i, j, k}$ of reorderings of a triple $(i, j, k)$ are related in the natural way.

- On 4 -fold intersections we require that $\delta \theta=1$, where $\delta$ is the Cech coboundary operator.

We refer to Ref. [24] for the notion of a refinement of a 2-gerbe and a proof of the fact that a 2 -gerbe naturally gives rise to a class in $H^{3}(X ; \mathbb{Z})$. Note that by passing to a sufficiently fine cover, the $\Gamma_{j}^{i}$ could be trivialized. Then, the above definition reduces to that of a Cech 3-cocycle. However, the above definition works for any open cover.

\footnotetext{
${ }^{27}$ The word "gerbe" in the following always refers to gerbes in the sense of Ref. [23] i.e., strict gerbes in the sense of Brylinski.

${ }^{28}$ We follow Ref. [24] Definition 2.1.1, here. These are termed "gerbs" in Ref. [24] and are shown to be identical with 2-gerbes in the sense of Ref. [23] later on in that paper.
} 
Note that the above definition of a 2-gerbe may be used to construct a continuous-trace algebra on $X$. The vector bundles $\Gamma_{j}^{i}$ give maps from $U_{i, j}$ to $\mathcal{P} U$. Since $\mathcal{P} U$ is isomorphic to $\operatorname{Aut}(\mathcal{K})$, these maps may be used to glue $C_{0}\left(U_{i}, \mathcal{K}\right)$ together along the $U_{i, j}$ to get a continuous-trace algebra as in Ref. [13]. Conversely, given a continuous-trace algebra on $X$, we obtain a gerbe, since the $\mathcal{P} U$ cocyles defining the continuous-trace algebra will give the vector bundles $\Gamma_{j}^{i}$. The remaining conditions are automatically satisfied, by definition. In particular, the image of the cohomology class of $\theta_{i, j, k}$ via the Bockstein map will be the Dixmier-Douady invariant.

Definition 4.2. A locally trivialized 3 -gerbe ${ }^{29}$ on a space $X$ consists of the following data:

- An open cover of $X$

$$
\left\{U_{i}: i \in I\right\} \quad \text { with } \bigcup_{I} U_{i}=X
$$

(and we write $U_{i j}=U_{i} \cap U_{j}$ and so on for other tuples of indices).

- A 2-gerbe, i.e., a continuous-trace algebra $\mathcal{A}_{i}^{j}$ over $U_{i j}$ for each ordered pair $(i, j), i \neq j$, such that the classes of $\mathcal{A}_{i}^{j}$ and $\mathcal{A}_{j}^{i}$ in $H^{3}\left(U_{i j}, \mathbb{Z}\right)$ are inverses of each other.

- A canonical trivialization $\Gamma_{i j k}$ of the tensor product $\left.\left.\mathcal{A}_{i}^{j}\right|_{U_{i j k}} \otimes \mathcal{A}_{j}^{k}\right|_{U_{i j k}} \otimes$ $\left.\mathcal{A}_{k}^{i}\right|_{U_{i j k}}$ (This would be a line bundle.) The line bundles $\Gamma$ are related in the natural way under reorderings of $(i, j, k)$.

- A trivialization of the coboundary of the $\Gamma_{i j k}$ on 4 -fold intersections $U_{i j k l}$, i.e., a canonical section $\eta_{i j k l}$ of

$$
\left.\left.\left.\left.\Gamma_{i j k}\right|_{U_{i j k l}} \otimes \Gamma_{i j l}^{-1}\right|_{U_{i j k l}} \otimes \Gamma_{i k l}\right|_{U_{i j k l}} \otimes \Gamma_{j k l}^{-1}\right|_{U_{i j k l}}
$$

and all the sections $\eta$ are related in the natural way under reorderings of $(i, j, k, l)$

- On 5 -fold intersections, we require that $\delta \eta=1$, where $\delta$ is the Cech coboundary operator.

The characteristic class of this 3 -gerbe is the cohomology class of $\eta \in$ $H^{4}(X ; \mathbb{Z})$. (Thus a 3 -gerbe would define a cohomology class in $H^{4}(X ; \mathbb{Z})$ in exactly a similar manner as a 2 -gerbe determines a cohomology class in $H^{3}(X ; \mathbb{Z})$.) From the above definitions, we can see that a $k$-gerbe possesses $(k-1)$-gerbes as "local sections". For example, a non-trivial line bundle (a 1-gerbe) has continuous functions as local non-zero sections. Similarly, a continuous-trace algebra (a 2-gerbe) has local objects which are line bundles. This may be seen as follows. Continuous-trace algebras satisfy Fell's condition, which guarantees the existence of a local rank-one projection in some

\footnotetext{
${ }^{29}$ We follow Ref. [24] Section 4.5 here.
} 
neighborhood $U_{x}$ of each $x \in X$. This is the same as a map $U_{x} \rightarrow \operatorname{Gr}\left(1, \mathcal{H}_{x}\right)$ for some Hilbert space $\mathcal{H}_{x}$. However, $\operatorname{Gr}\left(1, \mathcal{H}_{x}\right)$ is the classifying space for line bundles over $U_{x}$. The algebra is trivial if and only if there is a global rank-one projection. This would be the same as specifying a global line bundle on $X$ which would be the analogue, for a 2-gerbe, of a global section of a line bundle. Similarly, a 3-gerbe on $X$ (which would be classified by an element of $H^{4}(X ; \mathbb{Z})$ ) would have stable continuous-trace algebras as local sections. In the case of a line bundle, it is impossible to pick a global nonzero section unless the bundle is trivial, similarly, for a gerbe it is impossible to pick a global object, unless the gerbe is trivial. Hence, 2-gerbes may be used to study situations in which we have "partially defined" line bundles. This is the case, for example, in the monopole of the previous section. It also explains why we could naturally associate to it a class in $H^{3}$. Similarly, we expect 3 -gerbes to be useful for describing situations where we have "partially defined" continuous-trace algebras that is, sources of $H$-flux.

\subsection{Application to T-duality}

We consider a special case of T-duality formulated in Ref. [3], Lemma 4.5. Continuous-trace algebras on $B \times S^{1}$ are T-dual to $U(1)$-bundles $E$ on $B$. We may restate this by saying that T-duality gives a correspondence between 1-gerbes on $B$ and 2-gerbes on $B \times S^{1}$. If the characteristic class of the bundle $E$ is $[E]$, the $H$-flux of the T-dual is given by $[E] \times z$, where $z$ is the canonical generator of $H^{1}\left(S^{1}\right)$.

We would like to extend this correspondence to semi-free $S^{1}$-actions. A semi-free space $X$ is completely specified by the fixed point set $F \subset B$ and the class of the principal $S^{1}$-bundle over $B-F$. Thus, it is specified by a class $\lambda$ in $H^{2}(B-F)$.

This class may be used to construct a 2 -gerbe on $B^{+}$by taking the image of $\lambda$ in $H^{3}\left(B^{+}\right)$by the following sequence ${ }^{30}$ (see the previous section):

$$
H^{2}(B-F) \longrightarrow H^{3}(B, B-F) \longrightarrow H^{3}\left(B^{+}, B^{+}-F\right) \longrightarrow H^{3}\left(B^{+}\right) .
$$

The T-dual of such a space is, by the argument presented in Chapter 1, the space $B \times S^{1}$ with a source of $H$-flux located at $F \times S^{1}$. Such a source emits a $H$-flux which defines a class $\lambda \times z$ in $H^{3}\left((B-F) \times S^{1}\right)$.

\footnotetext{
${ }^{30}$ This sequence is not exact at $H^{3}(B, B-F)$ otherwise this class would always be zero.
} 
This class may be used to construct a 3 -gerbe on $B^{+} \times S^{1}$ by taking the image of $\lambda \times z$ in $H^{3}\left(B^{+}\right)$via the following sequence ${ }^{31}$ (see the previous section):

$$
\begin{aligned}
H^{3}\left((B-F) \times S^{1}\right) & \longrightarrow H^{4}\left(B \times S^{1},(B-F) \times S^{1}\right) \\
& \longrightarrow H^{4}\left(B^{+} \times S^{1},\left(B^{+}-F\right) \times S^{1}\right) \longrightarrow H^{4}\left(B^{+} \times S^{1}\right) .
\end{aligned}
$$

Thus, there seems to be a map between 2-gerbes on $B^{+}$and 3-gerbes on $B^{+} \times S^{1}$ induced by T-duality. This may be understood as follows. If we fix a generator $z$ of $H^{1}\left(S^{1}\right)$, then taking the cross product of a cohomology class $\lambda \in H^{k}(X)$ with $z$ gives a homomorphism $\times: H^{k}(X) \rightarrow H^{k+1}\left(X \times S^{1}\right)$. If $k=2$, this homomorphism is exactly the one which is induced by sending the characteristic class of a principal $S^{1}$ bundle to the H-flux on the T-dual trivial bundle. It is interesting therefore, that the same map for $k=3$ is also induced by T-duality.

Theorem 4.3. Let $X$ be a 2-gerbe on $B^{+}$with characteristic class $\eta \in$ $H^{3}\left(B^{+}\right)$. T-duality defines a map which sends $X$ to a 3 -gerbe $Y$ on $B^{+} \times S^{1}$ with characteristic class $(\eta \times z) \in H^{4}\left(B^{+} \times S^{1}\right)$.

Proof. Pick an open cover $\left\{U_{i}\right\}$ for $B^{+}$. Then, the 2-gerbe on $B^{+}$induces principal $S^{1}$-bundles $p_{i j}: L_{i}^{j} \rightarrow U_{i j}$. Let $\left[p_{i j}\right]$ denote the characteristic class of $L_{j}^{i}$. By the definition of a 2-gerbe (see Definition 4.1.), on $U_{i j k}$ the bundle

$$
\left.\left.\left.L_{i}^{j}\right|_{U_{i j k}} \otimes L_{j}^{k}\right|_{U_{i j k}} \otimes L_{k}^{i}\right|_{U_{i j k}}
$$

is trivial with a canonical section $\theta_{i j k}$. The definition requires $\delta \theta=1$. We take the cohomology class of $\theta$ to be $\eta$. T-dualizing each of the bundles $L_{j}^{i}$ gives continuous-trace algebras $A_{j}^{i}$ on $U_{i j} \times S^{1}$ with characteristic class $\left[p_{i j}\right] \times z$. Note that the characteristic class of $A_{j}^{i}$ and $A_{i}^{j}$ are inverses of each other in $H^{3}\left(U_{i j}\right)$ since

$$
\left(\left[p_{i j}\right] \times z\right)+\left(\left[p_{j i}\right] \times z\right)=\left(\left[p_{i j}\right]+\left[p_{j i}\right]\right) \times z=0 .
$$

Let $w_{\alpha}: U_{i j k} \rightarrow U_{\alpha}, \alpha=i j, j k, k i$ denote the inclusion map. Then, since the tensor product in equation (4.8) is trivial, we see that

$$
w_{i j}^{*}\left(\left[p_{i j}\right]\right)+w_{j k}^{*}\left(\left[p_{j k}\right]\right)+w_{k i}^{*}\left(\left[p_{k i}\right]\right)=0 .
$$

Let us try to compute the characteristic class of the tensor product

$$
\left.\left.\left.\mathcal{A}_{i}^{j}\right|_{U_{i j k}} \otimes \mathcal{A}_{j}^{k}\right|_{U_{i j k}} \otimes \mathcal{A}_{k}^{i}\right|_{U_{i j k}} .
$$

\footnotetext{
${ }^{31}$ This sequence too is not exact at $H^{3}(B, B-F)$ otherwise this class would always be zero.
} 
This would be given by

$$
\left(w_{i j} \times 1\right)^{*}\left(\left[p_{i j}\right] \times z\right)+\left(w_{j k} \times 1\right)^{*}\left(\left[p_{j k}\right] \times z\right)+\left(w_{k i} \times 1\right)^{*}\left(\left[p_{k i}\right] \times z\right),
$$

where

$$
w_{\alpha} \times 1:\left(U_{i j k} \times S^{1}\right) \longrightarrow\left(U_{\alpha} \times S^{1}\right), \alpha=\{i j, j k, k i\},
$$

are the induced inclusion maps on the T-dual side. This may be simplified as follows

$$
\begin{aligned}
& \left(w_{i j} \times 1\right)^{*}\left(\left[p_{i j}\right] \times z\right)+\left(w_{j k} \times 1\right)^{*}\left(\left[p_{j k}\right] \times z\right)+\left(w_{k i} \times 1\right)^{*}\left(\left[p_{k i}\right] \times z\right) \\
& \quad=w_{i j}^{*}\left(\left[p_{i j}\right]\right) \times z+w_{j k}^{*}\left(\left[p_{j k}\right]\right) \times z+w_{k i}^{*}\left(\left[p_{k i}\right]\right) \times z \\
& \quad=\left(w_{i j}^{*}\left(\left[p_{i j}\right]\right)+w_{j k}^{*}\left(\left[p_{j k}\right]\right)+w_{k i}^{*}\left(\left[p_{k i}\right]\right)\right) \times z=0
\end{aligned}
$$

Thus, the continuous-trace algebra defined in equation (4.10) is trivial. Thus, it must possess a section ${ }^{32}$ which would be a line bundle $\Gamma_{i j k}$ over $U_{i j k} \times S^{1}$. To obtain this section, we note that $\theta_{i j k}$ defines an element $\left[\theta_{i j k}\right] \in H^{1}\left(U_{i j k} ; \mathbb{Z}\right)$ and so we obtain an element $\left(\left[\theta_{i j k}\right] \times z\right) \in H^{2}\left(U_{i j k} \times\right.$ $\left.S^{1} ; \mathbb{Z}\right)$ which defines $\Gamma_{i j k}$.

Now, by Definition (4.2), restricting these $\Gamma$ to $U_{i j k l}$ and calculating the tensor product

$$
\left.\left.\left.\left.\Gamma_{i j k}\right|_{U_{i j k l}} \otimes \Gamma_{i j l}^{-1}\right|_{U_{i j k l}} \otimes \Gamma_{i k l}\right|_{U_{i j k l}} \otimes \Gamma_{j k l}^{-1}\right|_{U_{i j k l}}
$$

should give us a trivial bundle and a canonical section $\eta_{i j k l}$ which is a Cech cocycle. To show that the tensor product equation (4.12) is trivial, we once again calculate the characteristic class of this tensor product line bundle. If $w_{\alpha}: U_{i j k l} \rightarrow U_{\alpha}, \alpha=\{i j k, i j l, i k l, j k l\}$ is the inclusion map, the class we want to calculate is

$$
\begin{aligned}
& \left(w_{i j k} \times 1\right)^{*}\left(\left[\theta_{i j k}\right] \times z\right)-\left(w_{i j l} \times 1\right)^{*}\left(\left[\theta_{i j l}\right] \times z\right)+\left(w_{i k l} \times 1\right)^{*}\left(\left[\theta_{i k l}\right] \times z\right) \\
& -\left(w_{j k l} \times 1\right)^{*}\left(\left[\theta_{j k l}\right] \times z\right)
\end{aligned}
$$

This may be simplified as follows

$$
\begin{aligned}
\left(w_{i j k}\right. & \times 1)^{*}\left(\left[\theta_{i j k}\right] \times z\right)-\left(w_{i j l} \times 1\right)^{*}\left(\left[\theta_{i j l}\right] \times z\right) \\
& +\left(w_{i k l} \times 1\right)^{*}\left(\left[\theta_{i k l}\right] \times z\right)-\left(w_{j k l} \times 1\right)^{*}\left(\left[\theta_{j k l}\right] \times z\right) \\
= & \left(w_{i j k}^{*}\left(\left[\theta_{i j k}\right]\right)-w_{i j l}^{*}\left(\left[\theta_{i j l}\right]\right)+w_{i k l}^{*}\left(\left[\theta_{i k l}\right]\right)-w_{j k l}^{*}\left(\left[\theta_{j k l}\right]\right)\right) \times z .
\end{aligned}
$$

The term in parenthesis in the last equation is the class in $H^{1}\left(U_{i j k l}\right)$ induced by $\delta \theta$. Since $\delta \theta=1$, the expression vanishes. Note that if we change $\theta$ by a coboundary, the $\Gamma$ will change, but the tensor product will still remain trivial as its characteristic class will shift by the class in $H^{1}\left(U_{i j k l}\right)$ of the coboundary of a coboundary.

\footnotetext{
${ }^{32}$ See Definition 4.2 .
} 
We now need a trivialization of this tensor product on 5 -fold intersections. This is given by any representative of the cross product cocycle $\theta \times z$ which gives a cocycle on 5 -fold intersections and so a $\mathbb{C}^{*}$-valued function on this space. Changing the cocycle within its cohomology class will not change the gerbe as the characteristic class of the gerbe will remain the same.

Changing the original cover $\left\{U_{i}\right\}$ will not affect the answer, as following the above construction through on the new cover will show. It is also clear that the characteristic class of the 3 -gerbe so constructed will be $\eta \times z$.

Now, if we are given a space with a semi-free $S^{1}$-action with fixed point set whose image is $F \subset B$, then, as argued above, we get a class in $H^{2}(B-F)$ and a gerbe on $B^{+}$. If we pick an open cover of $B^{+}$containing $B-F$ and $B-\{+\}$, under T-duality, we will obtain by the above theorem a 3 -gerbe on $B^{+} \times S^{1}$ whose restriction to $B-F$ is exactly the continuous-trace algebra which is the T-dual of the line bundle we had over $B-F$.

We saw above that a semi-free $S^{1}$-space $X$ with quotient space a (compact, closed, and connected) manifold $B$ is classified up to equivariant homeomorphism by the fixed point set $F \subset B$ and the characteristic class of the principal $S^{1}$-bundle $\left(X-\pi^{-1}(F)\right) \stackrel{\pi}{\rightarrow}(B-F)$. We now assume that $F$ is a smooth embedded submanifold of $B$. We associated to $X$ a cohomology class in $H^{3}(B, B-F)$ which gave us a class in $H^{3}\left(B^{+}, B^{+}-F\right)$ by the excision isomorphism and finally gave us a class in $H^{3}\left(B^{+}\right)$(using the long exact sequence of the pair $\left(B^{+}, B^{+}-F\right)$ ). However, we could have obtained a class in $H^{3}(\tilde{B})$ for any compactification $\tilde{B}$ of $B$. $\left(B^{+}\right.$is not always a manifold even if $B$ is, so in applications we might need to use another compactification $\tilde{B}$.)

Lemma 4.4. There is a space $Y$, a map $H^{3}(B, B-F) \rightarrow H^{3}(Y)$ together with a natural map $\phi: H^{3}(Y) \rightarrow H^{3}(\tilde{B})$ such that every map $H^{3}(B, B-$ $F) \rightarrow H^{3}(\tilde{B})$ factors through $\phi$.

Proof. This space may be constructed as follows. If $N(F)$ is a tubular neighborhood of $F$ in $B$, then, by the tubular neighborhood theorem, $N(F)$ is diffeomorphic to the normal bundle of $F$ in $B$. Let $D(F)$ be the closure of $N(F)$ in $B$, then, $D(F)$ is homeomorphic to a disc bundle over $F$. By excision, and homotopy,

$$
H^{k}(B, B-F) \simeq H^{k}(D(F), D(F)-F) \simeq H^{k}(D(F), S(F)),
$$

where $S(F)$ is the sphere bundle which is the boundary of the disc bundle $D(F)$. Now, $H^{k}(D(F), S(F)) \simeq H^{k}(D(F) / S(F)) \simeq H^{k}(\mathrm{TD}(F))$ where $\operatorname{TD}(F)$ is the Thom space of the disc bundle $D(F)$ (see Ref. [25, page 441 
for details]). Now, for any space $\tilde{B}$ containing $F$, there is a collapse map $\lambda: \tilde{B} \rightarrow \mathrm{TD}(F)$ obtained by collapsing everything outside $N(F) \subset B \subset \tilde{B}$ to a point. The following diagram commutes:

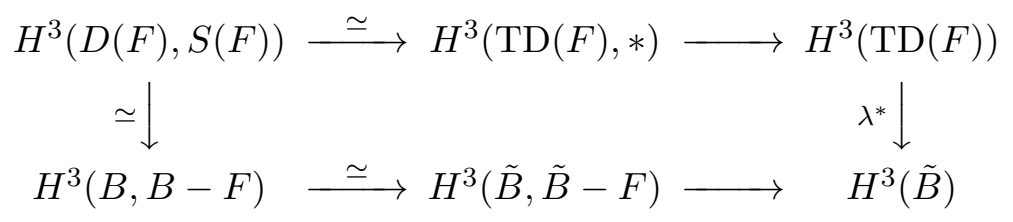

From this it follows that the image of any class in $H^{3}(\tilde{B})$ which is the image of a class $\gamma$ in $H^{3}(B, B-F)$ is actually pulled back from the image of $\gamma$ in $H^{3}(\operatorname{TD}(F))$ via $\lambda^{*}$. Thus, the image of $\gamma$ in $H^{3}(\operatorname{TD}(F))$ is a universal invariant.

It follows from this construction that the 2-gerbe we constructed in the previous subsection is actually pulled back from the 2-gerbe on $Y$ via the collapse map. It also follows from this construction that the invariant is zero once the codimension $k$ of $F$ is more than 3. For, by a property of the Thom space (See Ref. [25, page 441]), $H^{i}(\operatorname{TD}(F))=0$ if $i<k$. Further, we have the Thom isomorphism $\Phi: H^{i}(F) \rightarrow H^{i+k}(\operatorname{TD}(F))$. This should enable us to calculate the invariant explicitly.

On the T-dual side, we have a trivial $S^{1}$-bundle $B \times S^{1}$ with the NS5brane sitting somewhere in $F \times S^{1}$, transverse to the $S^{1}$-fiber. This would have a total charge given by a cohomology class in $H^{4}\left(\left(B \times S^{1}\right),(B-F) \times\right.$ $\left.S^{1}\right)$. By an argument similar to the above, we would have a commutative diagram.

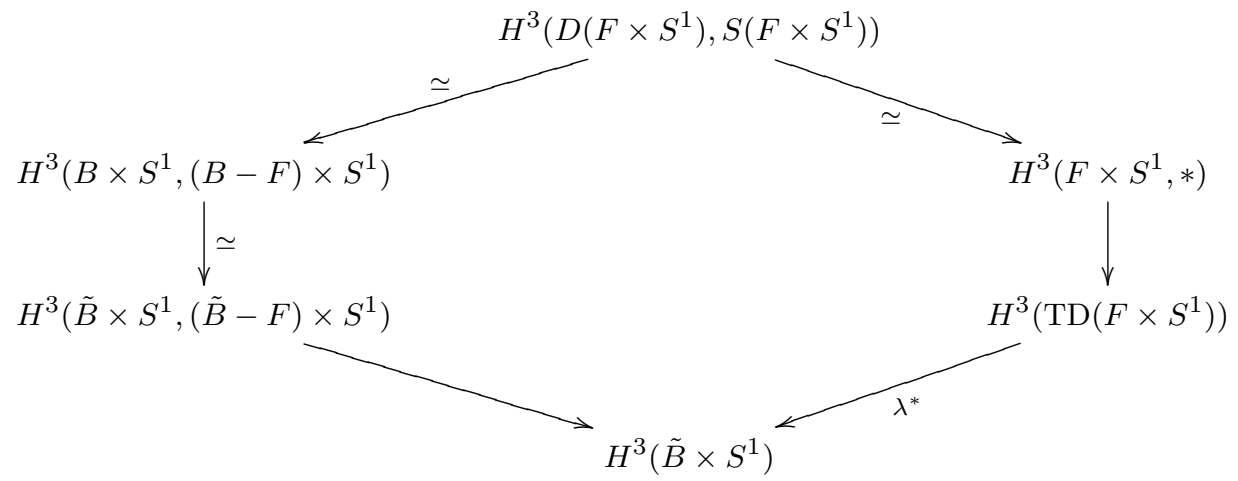

We also have a Thom isomorphism $\tilde{\Phi}: H^{i}\left(F \times S^{1}\right) \rightarrow H^{i+k}\left(\operatorname{TD}\left(F \times S^{1}\right)\right)$. Recall that T-duality gave a map $\times: H^{3}(B-F) \rightarrow H^{4}\left((B-F) \times S^{1}\right)$ given by the cross product with $z \in H^{1}\left(S^{1}\right)$. We saw in the previous section 
that this induced a map $\times: H^{3}\left(B^{+}\right) \rightarrow H^{3}\left(B^{+} \times S^{1}\right)$. An argument similar to the one given in that section would give also give a map $\times: H^{3}(\tilde{B}) \rightarrow$ $H^{4}\left(\tilde{B} \times S^{1}\right)$. Then we have a commutative diagram

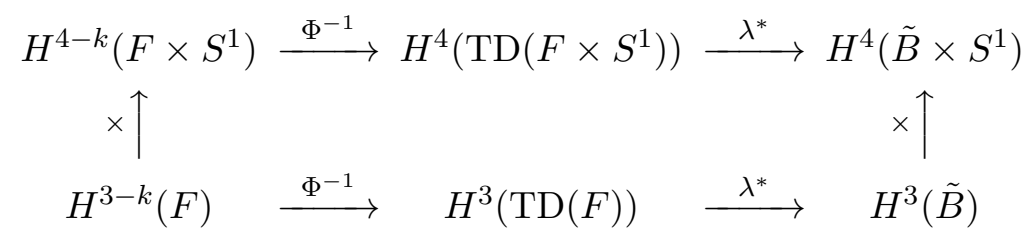

This may be used to calculate the invariant in $H^{4}$ for an NS5-brane configuration from the one in $H^{3}$.

\section{Conclusions and future work}

In this paper, we have studied the Topological T-dual of a semi-free $S^{1}$ space. We have shown how to model a wrapped NS5-brane within the formalism of Topological T-duality as an extension of $C^{*}$-algebras. We justify this by showing how it may be used to model the dyonic coordinate of a KK-monopole. We also study this extension using gerbes. We summarize the contents of this paper by the following commutative diagram. Each of the horizontal arrows is one of the results of this paper:

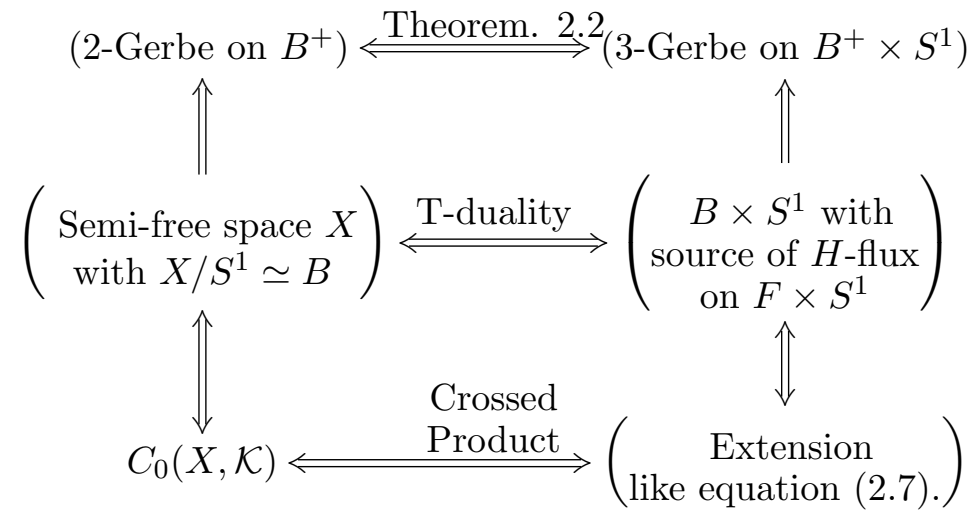

It would be interesting to extend this to semi-free $T^{n}$ spaces. It is shown in Ref. [3] that it may not always be possible to T-dualize such a space. However, it would be interesting to see if we could study the "brane box" [26] configurations using some extension of the present formalism. 
We showed using topological T-duality in Section 2 that the algebra obtained as the extension equation (2.7) is entirely determined by its spectrum, a cohomology class in $(X-F) / S^{1} \times S^{1}$, together with the fixed point set $F \subset X / S^{1}$. Is there an alternative characterisation of this $C^{*}$-algebra, independent of Topological T-duality? If so, this algebra would be a model for spaces with an arbitrary configuration of NS5-branes.

\section{Acknowledgments}

I thank Professor J. Rosenberg, University of Maryland, College Park, for key insights and helpful discussions. I also gratefully acknowledge the Research Assistantship which made this work possible. This Assistantship was supported by the NSF grant DMS-0504212.

\section{References}

[1] J. Polchinski, String theory, Cambridge University Press, 1998.

[2] E. Alvarez, L. Alvarez-Gaume, and Y. Lozano, An introduction to Tduality in string theory, Nucl. Phys. Proc. Suppl. 41 (1995), 1-20, also at hep-th/9410237.

[3] V. Mathai and J. Rosenberg, T-duality for torus bundles with H-fluxes via noncommutative topology, Comm. Math. Phys. 253 (2005), 705721.

[4] P. Bouwknegt, J. Evslin, and V. Mathai, T-duality: topology change from H-flux, Commun. Math. Phys. 249 (2004), 383-415, also at hepth/0306062.

[5] P. Bouwknegt, J. Evslin, and V. Mathai, On the topology and flux of T-dual manifolds, Phys. Rev. Lett. 92 (2004), 181601, also at hepth/0312052.

[6] I. Raeburn and J. Rosenberg, Crossed product of continuous-trace $C^{*}$-algebras by smooth actions, Trans. Amer. Math. Soc. 305(1) (1988), 1-45.

[7] G. Bredon, Introduction to compact transformation groups, Academic Press, 1972.

[8] B. Andreas, G. Curio, and D. Lust, The Neveu-Schwarz five-brane and its dual geometries, JHEP 9810 (1998), 022, also at hep-th/9807008.

[9] A.M. Uranga, Graduate course in string theory, Lecture 15, http:// gesalerico.ft.uam.es/paginaspersonales/angeluranga/firstpage.html.

[10] R. Sorkin, The Kauza-Klein monopole, Phys. Rev. Lett. 51(2), (1983), 87. 
[11] J.A. Harvey and S.A. Jensen, Worldsheet instanton corrections to the Kaluza-Klein monopole, JHEP 0510 (2005), 028, also at hepth/0507204.

[12] R. Gregory, J.A. Harvey and G. Moore, Unwinding strings and Tduality of Kaluza-Klein and H-monopoles, Adv. Theor. Math. Phys. 1 (1997), 283-297, also at hep-th/9708086.

[13] J. Rosenberg, Extensions of $C^{*}$-algebras, Proc. Symp. Pure Math. 38(I), AMS, 1992.

[14] U. Bunke and T. Schick, On the topology of T-duality, Rev. Math. Phys. 17(1) (2005), 77-112, also at math.GT/0405132.

[15] A. Sen, Kaluza-Klein dyons in string theory, Phys. Rev. Lett. 79 (1997), 1619.

[16] T. Hausel, E. Hunsicker and R. Mazzeo, Hodge cohomology of gravitational instantons, Duke Math. J. 122(3) (2004), 485-548, also at math.DG/0207169.

[17] J.A. Harvey, Magnetic monopoles, duality and supersymmetry, Report No. EFI-96-06, World Scientific, ICTP Series Theor. Phys. 12 (1997), 66.

[18] D. Crocker, A. Kumjian, I. Raeburn and D.P. Williams, An equivariant brauer group and actions of groups on $C^{*}$-algebras, J. Funct. Anal. 146(1) (1998), 151-184.

[19] I. Raeburn and D.P. Williams, Morita equivalence and continuous-trace $C^{*}$-algebras, Math. Surv. Monogr. 60, AMS, 1991.

[20] C.C. Moore, Group extensions and cohomology for locally compact groups III, Trans. Amer. Math. Soc. 221(1) (1976), 1-33.

[21] J.-L. Brylinski, Loop spaces, characteristic classes and geometric quantization, Birkhauser, Boston, 1993.

[22] G. Moore and E. Witten, Self-duality, Ramond-Ramond fields and K-theory, JHEP 0005 (2000), 032, also at hep-th/9912279.

[23] N. Hitchin, Lectures on special Lagrangian submanifolds, math.DG/ 9907034.

[24] D.S. Chatterjee, On the construction of Abelian Gerbs, Dissertation, University of Cambridge, 1998, also at http://www.ma. utexas.edu/ hausel/hitchin/hitchinstudents/chatterjee.pdf.

[25] A. Hatcher, Algebraic topology, Cambridge, Cambridge University Press, 2002.

[26] A.M. Uranga, Brane boxes and branes on singularities, JHEP, 9805 (1998), 013. 
